\title{
Review Article \\ Chinese Herbal Medicine as an Adjunctive Therapy for Breast Cancer: A Systematic Review and Meta-Analysis
}

\author{
Libing Zhu, Lingru Li, Yingshuai Li, Ji Wang, and Qi Wang \\ Center of Reproductive Medicine and Traditional Chinese Medicine Constitution, School of Preclinical Medicine, \\ Beijing University of Chinese Medicine, Beijing 100029, China
}

Correspondence should be addressed to Ji Wang; doctorwang2009@126.com and Qi Wang; wangqi710@126.com

Received 6 February 2016; Accepted 16 March 2016

Academic Editor: Oliver Micke

Copyright (c) 2016 Libing Zhu et al. This is an open access article distributed under the Creative Commons Attribution License, which permits unrestricted use, distribution, and reproduction in any medium, provided the original work is properly cited.

Chinese herbal medicine (CHM) has been widely used as an adjunctive therapy for breast cancer, while its efficacy remains unexplored. The purpose of this study is to evaluate the efficacy of CHM combined with chemotherapy for breast cancer. The study results showed that CHM combined with chemotherapy significantly increased tumor response and KPS as compared to using chemotherapy alone $(\mathrm{RR}=1.36 ; 95 \% \mathrm{CI}=1.24-1.48 ; P<0.00001 ; \mathrm{RR}=1.38 ; 95 \% \mathrm{CI}=1.26-1.52 ; P<0.00001$, resp. $)$. Besides, $\mathrm{CHM}$ as an adjunctive therapy significantly reduced the nausea and vomiting at toxicity grade of III-IV $(\mathrm{RR}=0.37 ; 95 \% \mathrm{CI}=0.27-$ $0.52 ; P<0.00001)$. Moreover, the combined therapy significantly prevented the decline of WBC in patients under chemotherapy at toxicity grade of III-IV $(\mathrm{RR}=0.49 ; 95 \% \mathrm{CI}=0.34-0.69 ; P<0.00001)$ and prevented the decline of platelet at toxicity grade of III-IV or I-IV $(\mathrm{RR}=0.29 ; 95 \% \mathrm{CI}=0.12-0.73 ; P=0.008 ; \mathrm{RR}=0.77 ; 95 \% \mathrm{CI}=0.63-0.94 ; P=0.009$, resp. $)$. This study suggests that $\mathrm{CHM}$ combined with chemotherapy in comparison with chemotherapy alone can significantly enhance tumor response, improve KPS, and alleviate toxicity induced by chemotherapy in breast cancer patients. However, a firm conclusion could not be reached due to the lack of high quality trials and large-scale RCTs, so further trials with higher quality and larger scale are needed.

\section{Introduction}

Breast cancer is the most common malignancy and the leading cause of cancer mortality in women worldwide [1]. American Cancer Society (ACS) estimates that there will be 246,660 cases of women diagnosed with breast cancer in US and 40,450 women die of the disease during 2016 [2]. The prognosis of newly diagnosed breast cancer patients is determined by the classification of breast cancer. There are at least four main subtypes of breast cancer according to different patterns of gene expression [3, 4]. Luminal A tends to have the best prognosis, which comprises estrogen receptor-positive (ER+) and/or progesterone receptorpositive $(\mathrm{PR}+)$, human epidermal growth factor receptor 2 negative (HER2-), and grade 1 or 2 tumors [3]. Luminal B includes ER+ and/or PR+, HER2+ or HER2-, and grade 3 tumors. The other 2 subtypes confer bad prognosis, which contain the HER2 overexpressing breast cancer (ER-, PR-, and HER2+) and the triple-negative breast cancer (ER-, PR-, and HER-) [4]. Nowadays the standard treatment options for patients with breast cancer include surgery, chemotherapy, radiotherapy, and endocrine therapy [5]. Usually, endocrine therapy remains the core adjuvant therapy for most of the early breast cancer patients who are diagnosed as ER+, while chemotherapy is recommended as the first-line systemic adjuvant modality for most HER $2+$ and triple-negative breast cancer patients [6]. Unfortunately, even curing surgery may accompany the risk of recurrence and metastasis, patients may produce resistance to chemotherapy, and these treatments can cause serious side effects in short or long term $[6,7]$.

Complementary and alternative medicine (CAM) is widely used by $50 \%$ cancer patients, and patients with breast cancer use CAM more frequently than others which is accounting for 63 to $83 \%$ [8-12]. The types of CAM used by patients with breast cancer vary between countries. In US and Europe, naturopathy and homeopathy are the most commonly used CAM types, whereas for patients with Chinese background, Chinese herbal medicine (CHM) tends to be the most popular type among cancer patients and $86.4 \%$ 
breast cancer patients used CHM for treatment $[5,9,13,14]$. $\mathrm{CHM}$ as an adjuvant therapy to conventional therapy (mainly refers to chemotherapy) has been commonly used to prolong survival time of cancer patients, alleviate clinical symptoms, and minimize the adverse events caused by chemotherapy in Asia [15]. Previous clinical studies suggested that CHM adjuvant therapy might have potential roles in three main parts. First of all, it can improve the quality of life (QoL) and prolong the survival time [16]. Next, it may improve the immune function of breast cancer patients and prevent metastasis and recurrence $[17,18]$. Finally, it can prevent or reduce toxicity from anticancer agents and enhance the effect of anticancer agents $[16,17]$. However, there is lack of systematic review to assess the potential efficacy of CHM as an adjuvant therapy for breast cancer. Thus, the aim of this study is to conduct a systematic review to evaluate the efficacy of CHM combined with chemotherapy for breast cancer, using evidence from randomized controlled trials (RCTs) upon tumor response, immune function, adverse events, and QoL.

\section{Methods}

RCTs were retrieved from nine databases, theses, and conference papers by using electronic method as well as manual method. LBZ and LRL reviewed these studies independently. The first reviewer extracted the data from included studies and the second reviewer verified them again. Two reviewers rectified the discrepancies according to the original articles. If the consensus still cannot be achieved, a third party (QW) were sought for advising. Studies can be included in the metaanalysis only when they satisfied the criteria.

2.1. Search Strategy. The following databases were retrieved without any language restriction: PubMed, Cochrane Central Register of Controlled Trials, ISI Web of Science, Scopus, CINAHL Plus (EBSCO), EMBASE, China Journal Full-Text Database, China National Knowledge Infrastructure (CNKI), and Chinese Scientific Journal Database. Publications available from the inception of databases to January 2016 were reviewed to find out the appropriate RCTs of CHM for breast cancer. The following terms were searched in the databases: (Traditional Chinese Medicine OR Chinese Medicine OR traditional herbal medicine OR Chinese herbal medicine OR Chinese herbal drug OR herbal medicine OR traditional Japanese medicine OR traditional medicine OR materia medica OR Oriental medicine OR herb OR medicinal plant OR medicinal herbs OR medicinal plant product OR plant extract OR plant preparation OR herbal preparation OR phytotherapy OR herb therapy OR alternative medicine OR alternative therapy OR complementary therapy OR complementary medicine OR complementary and alternative therapy) AND (breast cancer OR breast carcinoma OR mammary cancer OR breast tumor) AND (clinical trial OR phase 1 clinical trial OR phase 2 clinical trial OR phase 3 clinical trial OR phase 4 clinical trial OR controlled clinical trial OR randomized controlled trial). The above terms in Chinese were searched in Chinese databases.
2.2. Inclusion Criteria. Only studies which meet all of the following criteria can be included in the meta-analysis. (1) Participants: participants are postoperative breast cancer patients and patients treated by chemotherapy. (2) Type of study: only RCTs were eligible. (3) Type of intervention: studies compared chemotherapy combined with or without CHM. For studies using other agents as the third arm, only the two arms using CHM with or without chemotherapy can be included for meta-analysis. (4) Type of outcome measurement: tumor response and Karnofsky performance score (KPS) were the main outcome measurements; other outcome measurements which contained immunoregulation and reduction in adverse events of chemotherapy were also considered.

2.3. Exclusion Criteria. Studies were excluded if they did not meet the above inclusion criteria. Additionally, trials with one or more of the following conditions were also excluded: (1) nonoriginal research such as review articles or letter to the editors; (2) duplicated publications of other studies; (3) CHM which were used in both treatment group and control group.

2.4. Outcome Measures. Tumor response of CHM on the number of breast cancer patients with complete response (CR) or partial response (PR), as well as those with progressive disease (PD) according to the WHO scale, was investigated [19]. The disappearance of all known tumor lesions is considered as $\mathrm{CR}, 50 \%$ or more reduce in total tumor size of the lesions is considered as PR (determined by two observations not shorter than 4 weeks apart), and $\mathrm{PD}$ refers to $25 \%$ or more increase in total tumor size of the lesions or the appearance of new lesions. The improved or stable performance status of subjects based on KPS was also examined, in which score of 100 refers to a normal subject without any complaints, score of 70 refers to a patient who is unable to carry on normal activity, score of 50 refers to a patient requires considerable assistance, score of 40 refers to a disabled patient, and score of 30 refers to a patient who is hospitalization-recommended [20]. The efficacy of CHM on relieving the adverse events caused by chemotherapy containing nausea and vomiting and reduction of white blood cell as well as platelet were studied by grading the acute and subacute toxic effects of cancer therapy [21]. The efficacy of $\mathrm{CHM}$ on immunoregulation includes the change of mean values of CD3 T cell level, CD4 T cell level, CD8 T cell level, and $\mathrm{CD} 4 / \mathrm{CD} 8$ ratio.

2.5. Quality Assessment. Methodological quality of included studies was assessed by using the risk of bias tools in accordance with Cochrane Handbook version 5.1.0 [22]. Risk of bias for assessing the methodological quality of RCTs mainly included six items: selection bias (random sequence generation and allocation concealment), performance bias and detection bias (blinding), attrition bias (incomplete outcome data), reporting bias (selective reporting), and other bias. Each item was ranked as low, high, and unclear risk. The methodological quality of all trials was assessed as the following three levels: low risk of bias (all items were ranked 
as low risk), unclear risk of bias (at least one item was ranked as unclear risk), or high risk of bias (at least one item was ranked as high risk). At least two reviewers assessed the all trials and any disagreements were solved by the third reviewer consensus.

2.6. Data Analysis. Cochrane Collaboration Review Manage software (RevMan 5.2) was used for data analysis. Dichotomous data were reported as relative risk (RR) with 95\% confidence intervals ( $95 \% \mathrm{CI}$ ) whereas continuous data were expressed as mean \pm standard deviation (SD). $I^{2}$ was used to assess the heterogeneity; if the heterogeneity exists in the pooled studies $\left(I^{2}>50 \%\right)$, a random-effect model was applied; otherwise the fix-effect model was applied [23]. The differences between the treatment groups and control groups were considered to be statistically significant when $P<0.05$.

\section{Results}

3.1. Characteristics of the Included Studies. Overall, 571 studies were retrieved. There were 11 duplicated studies, 127 studies did not investigate outcome of interest (such as only investigated Chinese medicine syndrome scales), 29 studies were literature reviews, 26 studies were animal studies, 9 studies were mechanism studies, 157 were not relevant studies (such as investigated CHM for lymphedema after mastectomy), 50 studies were not using CHM combined with chemotherapy as intervention, and 8 studies were not RCTs. So only 154 studies satisfied the selection criteria, among which 106 studies did not investigate outcome of interest, 9 studies did not use CHM combined with chemotherapy as interventions, 1 study was not RCT, and 5 studies were with incomplete data. There were 33 RCTs included in this metaanalysis [16-18, 24-54] (Figure 1). A sum of 2098 patients was enrolled in these studies, at which 1066 patients participated in CHM combined chemotherapy and 1032 in chemotherapy (two patients dropped out from treatment group, one patient dropped out from control group, and other four patients withdraw or dropped out but did not report the specific number in each group). All patients recruited in the 33 studies were postoperative breast cancer patients, and basically all of the included studies can be evaluated as low risk of bias [16-18, 24-54]. The risk of bias of all included studies was shown in Figures 2 and 3. The course of therapy varied from 2 to 24 weeks in included studies. A list of therapeutic approaches and outcome measurements in each study were listed in Table 1. All studies claimed that the baseline data were comparable containing age, TNM (tumor node metastasis) stage, or histopathology.

3.2. Tumor Response. Results from 19 studies showed that $69 \%(466 / 671)$ of patients using chemotherapy with CHM were reported as complete or partial response, while $51 \%$ (324/633) of patients only using chemotherapy were reported as complete or partial response, indicating that the treatment for breast cancer was significantly in favour of CHM combined with chemotherapy $(\mathrm{RR}=1.36$; $95 \% \mathrm{CI}=1.24-$ $1.48 ; P<0.00001)[24,26,28-31,33,36-38,40-43,46-50]$ (Figure $4(\mathrm{a})) .7 \%(47 / 671)$ of patients in the chemotherapy combined with CHM group were reported with progressive disease, while 16\% (100/633) of patients without CHM were reported with progressive disease. Results from 19 studies showed that the combined treatment for breast cancer has a positive effect in the number of patients who reported progressive disease $(\mathrm{RR}=0.45 ; 95 \% \mathrm{CI}=0.33-0.62 ; P<0.00001)$ $[24,26,28-31,33,36-38,40-43,46-50]$ (Figure 4(b)).

3.3. Performance Status. The QoL changes on KPS were reported as two types of data in the included studies, the number of patients who reported the improved or stable performance status based on KPS (ten-point cutoff) and the mean \pm SD of KPS before and after treatment. For the nondeterioration KPS, 11 studies of the 33 studies with evaluation of 634 patients were analyzed. There were $87 \%$ $(283 / 325)$ of patients who reported nondeterioration in the combined therapy groups and in the chemotherapy groups it was $63 \%(195 / 309)[18,27,28,34,39,40,44,45,48$, 50, 53]. Results from 11 studies showed that the combined using of chemotherapy and CHM was significantly related to improving QoL $(\mathrm{RR}=1.38 ; 95 \% \mathrm{CI}=1.26-1.52 ; P<0.00001)$ (Figure 5(a)). There was no significant heterogeneity among these studies $\left(I^{2}=0 \%\right)$. The value of KPS was reported with pretreatment in seven studies [24, 25, 32, 38, 41, 42, 54] and posttreatment in eight studies $[24,25,32,33,38,41$, $42,54]$, and the pooled studies of pretreatment showed that there was no significant difference between combined therapy and chemotherapy alone $(\mathrm{SMD}=0.18 ; 95 \% \mathrm{CI}=-0.02-$ $\left.0.39 ; P=0.07 ; I^{2}=0 \%\right)$. However, the pooled studies of posttreatment indicated significant difference between CHM combined with chemotherapy and chemotherapy alone $\left(\mathrm{SMD}=1.32 ; 95 \% \mathrm{CI}=0.99-1.65 ; P<0.00001 ; I^{2}=59 \%\right)$ (Figure 5(b)). Although $I^{2}$ is equal to $59 \%$, dropping one of any of the studies did not change the result in favour of combined therapy.

3.4. Reduction in Chemotherapeutic Toxicity. Nausea and vomiting are common adverse events of chemotherapy. There was significant reduction of nausea and vomiting at toxicity grade of III-IV in patients treated by CHM combined with chemotherapy $(\mathrm{RR}=0.37 ; 95 \% \mathrm{CI}=0.27-0.52 ; P<0.00001$; twelve studies; 694 patients) $[25,27,28,35,41,43,46$, 48-50, 52, 53] (Figure 6(a)). However, the reduction of nausea and vomiting at toxicity grade of I-IV has significant heterogeneity $(\mathrm{RR}=0.75 ; 95 \% \mathrm{CI}=0.69-0.82 ; P<0.00001$; $I^{2}=91 \%$ ) (data not shown). A significant reduction of WBC inhibition at toxicity grade of III-IV was found $(\mathrm{RR}=$ 0.49; 95\% CI $=0.34-0.69 ; P<0.00001$; twelve studies; 690 patients) $[16,25,27,35,41,43,46,48-50,52,54]$ (Figure 6(b)). But there was significant heterogeneity in the studies with reduction of WBC inhibition at toxicity grade of $\mathrm{I}-\mathrm{IV}(\mathrm{RR}=$ $0.75 ; 95 \% \mathrm{CI}=0.69-0.82 ; P<0.00001 ; I^{2}=91 \%$ ) (data not shown). The decrease of platelet at the toxicity grade of III-IV or I-IV in patients with combined therapy was significantly reduced $(\mathrm{RR}=0.29 ; 95 \% \mathrm{CI}=0.12-0.73 ; P=0.008$; seven studies; 453 patients; $\mathrm{RR}=0.77 ; 95 \% \mathrm{CI}=0.63-0.94 ; P=$ 0.009; seven studies; 453 patients, resp.) (Figures 6(c) and $6(d))[16,25,35,41,48,52,53]$. 


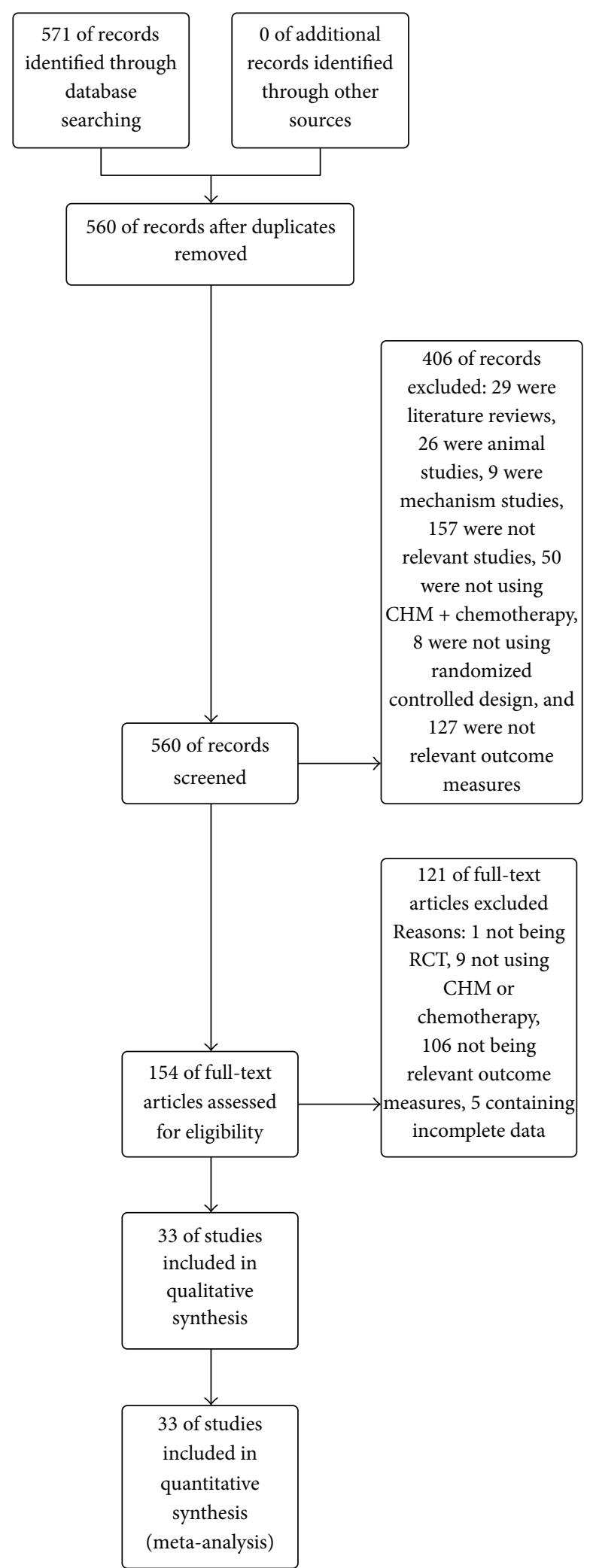

FIgURE 1: Study flow diagram. 


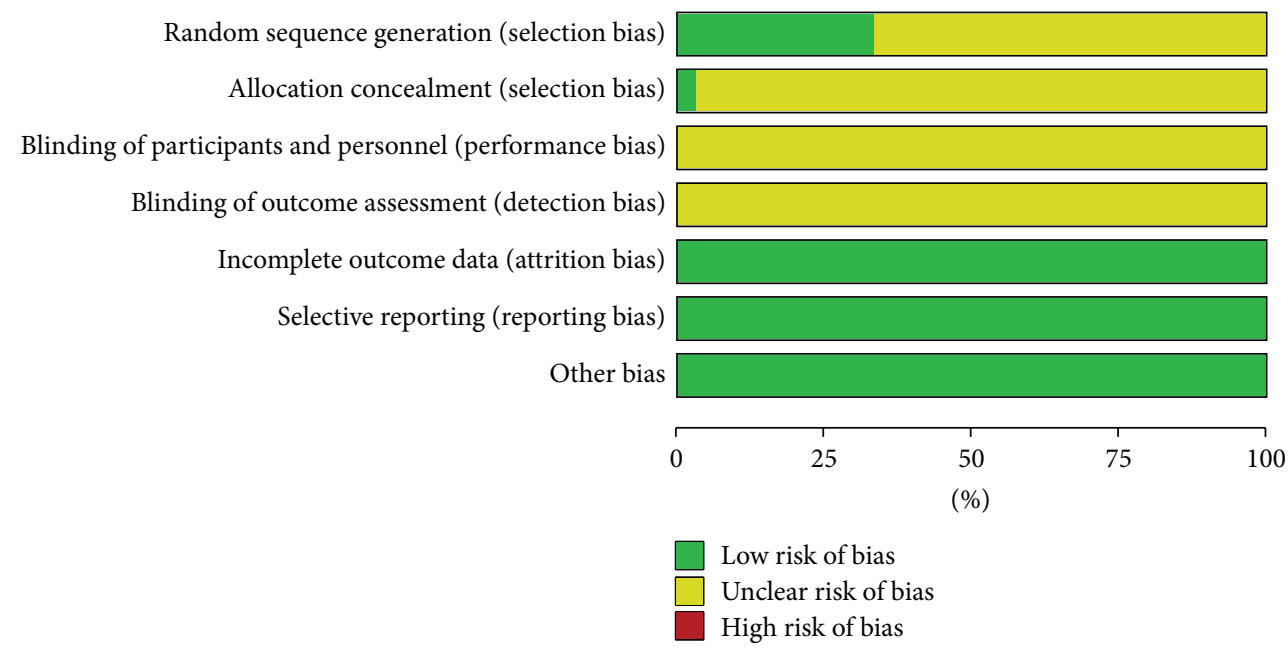

FIgURE 2: Risk of bias graph.

3.5. Immunoregulation. There was a significant rise in CD3 T cell level in patients treated by chemotherapy combined with CHM. However, a significant difference of the heterogeneity test was found among the pooled seven studies $(\mathrm{MD}=5.85$; 95\% CI = 3.72-7.98; $P<0.00001 ; I^{2}=68 \%$ ) (Figure 7(a)) $[16$, $25,38,39,45,48,54]$. In addition, significant improvement in other immune effects also occurred in combined therapy group, including CD4 T cell level $(\mathrm{MD}=5.27$; 95\% CI $=3.03-$ 7.51; $P<0.00001$; nine studies; 555 patients) (Figure $7(\mathrm{~b})$ ) $[16,17,25,38,39,44,45,48,54]$, CD8 T cell level $(\mathrm{MD}=$ -3.93; 95\% CI $=-6.04--1.82 ; P=0.0003$; eight studies; 498 patients) (Figure $7(\mathrm{c}))[14,17,38,39,44,45,48,54]$, and $\mathrm{CD} 4 / \mathrm{CD} 8$ ratio $(\mathrm{MD}=0.27 ; 95 \% \mathrm{CI}=0.15-0.40 ; P<0.0001$; eight studies; 505 patients) (Figure $7(\mathrm{~d})$ ) [16, 17, 25, 39, 44, 45, $48,54]$. However, the heterogeneity test of CD4 T cell level $\left(I^{2}=87 \%\right)$, CD8 T cell level $\left(I^{2}=85 \%\right)$, and CD4/CD8 ratio $\left(I^{2}=67 \%\right)$ indicated significant difference among these studies. Interestingly, these studies all claimed that significant improvement was found in CHM and chemotherapy as compared with chemotherapy alone.

3.6. Herbs Frequently Used in Breast Cancer. 30 studies reported herbs and decoctions. Among them, Radix Astragalus, Rhizoma Atractylodis Macrocephalae, and Angelica sinensis are the most frequently used herbs for breast cancer (Table 2).

\section{Discussion}

Recent studies showed a high prevalence of CAM usage among cancer patients, in particular patients with breast cancer $[5,8,9,12,14,55]$. CHM is an especially popular CAM used for cancer patients, while the efficacy of CHM combined with chemotherapy for patients under breast cancer remains unknown and needs to be further explored due to the language barrier because many studies were published in Chinese language. This study conducted a metaanalysis to statistical analysis of the results from individual studies for the purpose of integrating the findings. In the study, the pooled data has shown that CHM combined with chemotherapy significantly improved the tumor response and performance status of breast cancer patients. Also, we found that the combined therapy significantly decreases adverse events caused by chemotherapeutic interventions as compared with chemotherapy alone, including nausea and vomiting at toxicity grade of III-IV, WBC reduction at toxicity grade of III-IV, and platelet reduction at toxicity grade of IIV or III-IV (Figures 6(a), 6(b), 6(c), and 6(d)). The efficacy of $\mathrm{CHM}$ as an adjuvant therapy to chemotherapy for breast cancer is in line with the findings from meta-analysis of CHM combined therapy for advanced non-small-cell lung cancer, colorectal cancer, nasopharyngeal carcinoma, and hepatocellular carcinoma, which suggest that chemotherapy combined with CHM has an advantage in various cancers [20, 56-58].

According to Traditional Chinese Medicine (TCM) theory, sickness is caused by the imbalance of Yin and Yang; restoring the balance of Yin and Yang is the key for curing disease. People can have powerful self-healing ability to remove pathogenic factors and regain health when Yin and Yang of human body are balanced. And self-healing power of human body is attached to importance in the treatment of cancer [59]. Radix Astragalus (73\%), Rhizoma Atractylodis Macrocephalae (61\%), Angelica sinensis (48\%), Codonopsis pilosula (45\%), and Poria cocos (39\%) were identified as the top five frequently used herbs in the study; all of them have the function of tonifying Qi except Angelica sinensis have the function of enriching blood and promoting blood flow, which are in line with the commonest symptoms in postoperative breast cancer patients who are undergoing chemotherapy (such as Qi-deficiency, Qi-blood-deficiency, or bloodstasis). For the most frequently used herb Radix Astragalus, some studies have demonstrated that it has the efficacy of antitumor, immunoregulation, and immune restoration by stimulating macrophage and natural killer cell activity while inhibiting T-helper cell type 2 cytokines [60-62]. Besides, Radix Astragalus can reduce the toxicity induced by 


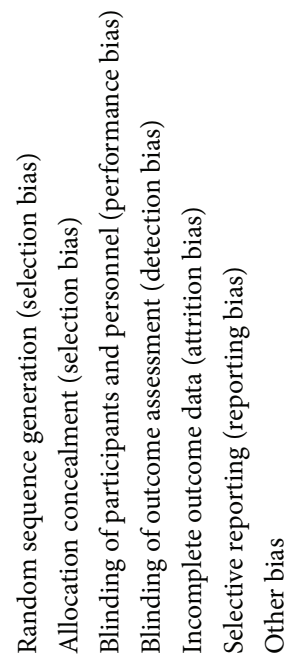

Bai and Wu 2014

Chen et al. 2008

Chen et al. 2015

Cheng 2011

\begin{tabular}{|l|l|l|l|l|l|l|}
\hline$?$ & $?$ & $?$ & $?$ & $\oplus$ & $\oplus$ & $\oplus$ \\
\hline
\end{tabular}

\begin{tabular}{|c|c|c|c|c|c|c|}
\hline & $?$ & $?$ & $?$ & $\oplus$ & $\oplus$ & $\oplus$ \\
\hline
\end{tabular}

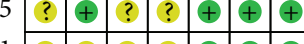

Cheng et al. 2013

Dai et al. 2004

\begin{tabular}{lllllllll}
$?$ & $?$ & $?$ & $?$ & $\oplus$ & $\oplus$ & $\oplus$ \\
\hline
\end{tabular}

Du et al. 2015

Fang and Jia 2015

Feng et al. 2010

Guo 2014

Hu et al. 2008

Huang et al. 2003

Huang et al. 2007

Li et al. 2004

Li 2015

Li and Han 2015

Liang et al. 2015

J. Liu et al. 2011

C. Liu et al. 2011

Pan 2011

Tang et al. 2007

Wang 2013

Wang 2015

Xie 2014

Xiong 2012

Yang and Sun 2004

Yang et al. 2007

Yang et al. 2008

Yang 2011

Yang et al. 2014

Zhang 2009

Zhang et al. 2011

Zhang and Dang 2012

\begin{tabular}{lllllll|l|l|}
\hline & $?$ & $?$ & $?$ & $\oplus$ & $\oplus$ & $\oplus$ \\
\hline
\end{tabular}

\begin{tabular}{|l|l|l|l|l|l|l|}
\hline$?$ & $?$ & $?$ & $?$ & $\oplus$ & $\oplus$ & $\oplus$ \\
\hline
\end{tabular}

\begin{tabular}{|l|l|l|l|l|l|l|}
\hline$?$ & $?$ & $?$ & $?$ & $\oplus$ & $\oplus$ & $\oplus$ \\
\hline
\end{tabular}

\begin{tabular}{lllllllll}
$?$ & $?$ & $?$ & $?$ & $\oplus$ & $\oplus$ & $\oplus$ \\
\hline
\end{tabular}

\begin{tabular}{|l|l|l|l|l|l|l|}
\hline$?$ & $?$ & $?$ & $?$ & $\oplus$ & $\oplus$ & $\oplus$ \\
\hline
\end{tabular}

\begin{tabular}{|l|l|l|l|l|l|l|}
\hline$?$ & $?$ & $?$ & $?$ & $\oplus$ & $\oplus$ & $\oplus$ \\
\hline
\end{tabular}

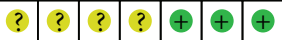

\begin{tabular}{lllll|lll}
$\oplus$ & $?$ & $?$ & $?$ & $\oplus$ & $\oplus$ & $\oplus$ \\
\hline
\end{tabular}

\begin{tabular}{c|c|c|c|c|c|c|}
+ & $?$ & $?$ & $?$ & $\oplus$ & $\oplus$ & $\oplus$ \\
\hline
\end{tabular}

\begin{tabular}{llllllll}
$?$ & $?$ & $?$ & $?$ & $\oplus$ & $\oplus$ & $\oplus$ \\
\hline
\end{tabular}

\begin{tabular}{llllllllll}
\hline & $?$ & $?$ & $?$ & $\oplus$ & $\oplus$ & $\oplus$ \\
\hline
\end{tabular}

\begin{tabular}{|l|l|l|l|l|l|l|}
\hline & $?$ & $?$ & $?$ & $\oplus$ & $\oplus$ & $\oplus$ \\
\hline
\end{tabular}

\begin{tabular}{|l|l|l|l|l|l|l|}
\hline & $?$ & $?$ & $?$ & $\oplus$ & $\oplus$ & $\oplus$ \\
\hline
\end{tabular}

\begin{tabular}{|l|l|l|l|l|l|l|}
\hline$?$ & $?$ & $?$ & $?$ & $\oplus$ & $\oplus$ & $\oplus$ \\
\hline
\end{tabular}

\begin{tabular}{llllllll|l}
\hline$?$ & $?$ & $?$ & $?$ & $\oplus$ & $\oplus$ & $\oplus$ \\
\hline
\end{tabular}

\begin{tabular}{llllll|l|l|}
\hline$?$ & $?$ & $?$ & $?$ & $\oplus$ & $\oplus$ & $\oplus$ \\
\hline
\end{tabular}

\begin{tabular}{lllllllll}
$?$ & $?$ & $?$ & $?$ & $\oplus$ & $\oplus$ & $\oplus$ \\
\hline
\end{tabular}

\begin{tabular}{llllllllll}
\hline$?$ & $?$ & $?$ & $?$ & $\oplus$ & $\oplus$ & $\oplus$ \\
\hline
\end{tabular}

\begin{tabular}{lllllllll}
\hline$?$ & $?$ & $?$ & $?$ & $\oplus$ & $\oplus$ & $\oplus$ \\
\hline
\end{tabular}

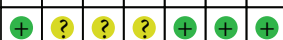

\begin{tabular}{lllllllll}
\hline & $?$ & $?$ & $?$ & $\oplus$ & $\oplus$ & $\oplus$ \\
\hline
\end{tabular}

\begin{tabular}{|l|l|l|l|l|l|l|}
\hline$?$ & $?$ & $?$ & $?$ & $\oplus$ & $\oplus$ & $\oplus$ \\
\hline
\end{tabular}

\begin{tabular}{lll|l|l|l|l|l|}
\hline$?$ & $?$ & $?$ & $?$ & $\oplus$ & $\oplus$ & $\oplus$ \\
\hline
\end{tabular}

\begin{tabular}{|c|c|c|c|c|c|c|}
\hline$\oplus$ & $?$ & $?$ & $?$ & $\oplus$ & $\oplus$ & $\oplus$ \\
\hline
\end{tabular}

\begin{tabular}{llllll|l|l|}
$?$ & $?$ & $?$ & $?$ & $\oplus$ & $\oplus$ & $\oplus$ \\
\hline
\end{tabular}

$\begin{array}{lllllllll}? & ? & ? & ? & \oplus & \oplus & \oplus\end{array}$

\begin{tabular}{lllllllll}
$?$ & $?$ & $?$ & $?$ & $\oplus$ & $\oplus$ & $\oplus$ \\
\hline
\end{tabular}

\begin{tabular}{|l|l|l|l|l|l|l|}
\hline+ & $?$ & $?$ & $?$ & $\oplus$ & $\oplus$ & $\oplus$ \\
\hline
\end{tabular}

\begin{tabular}{ll|l|l|l|l|l}
\hline$?$ & $?$ & $?$ & $?$ & $\oplus$ & $\oplus$ & + \\
\hline
\end{tabular}

FIGURE 3: Risk of bias summary. 


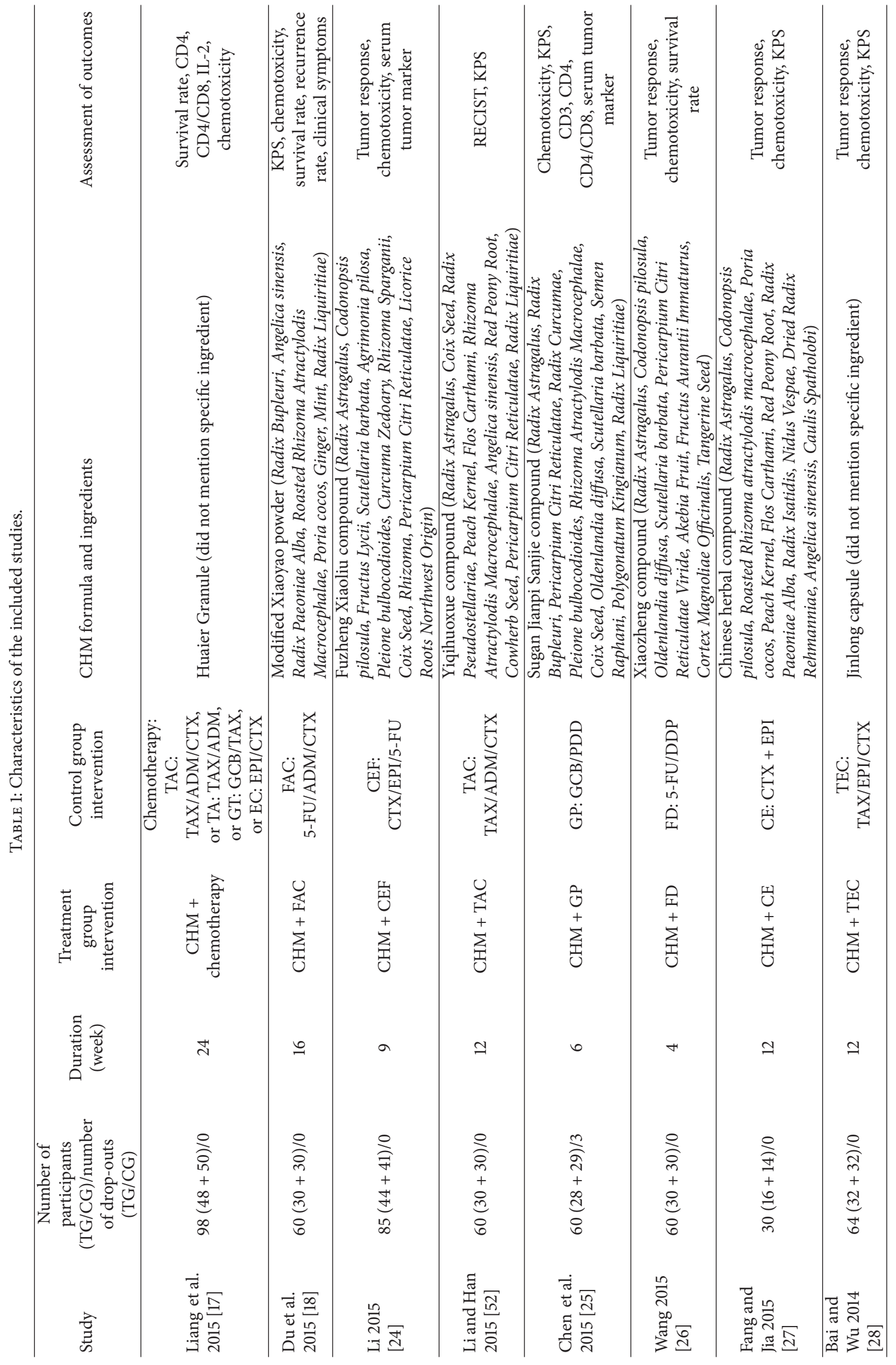




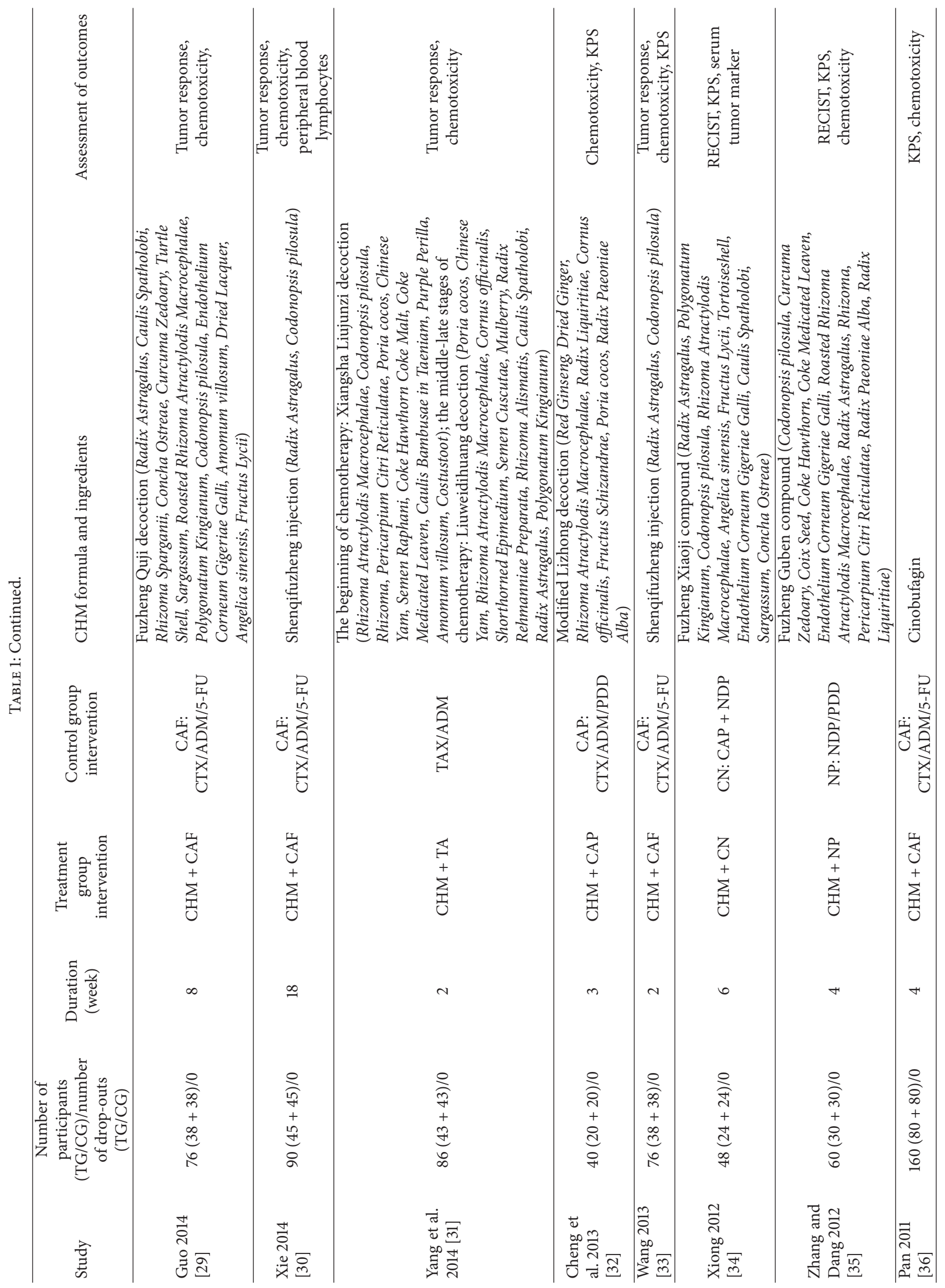




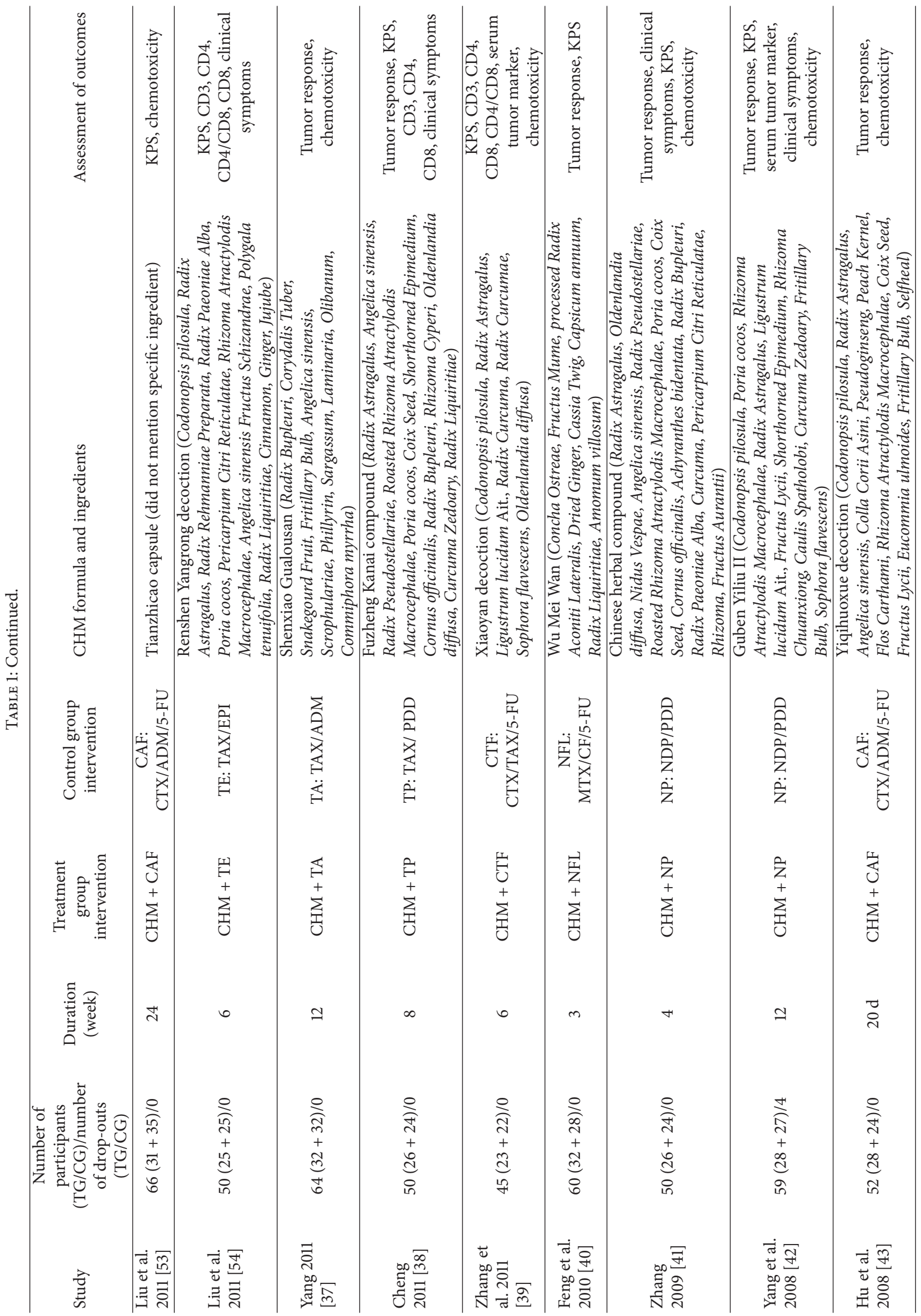




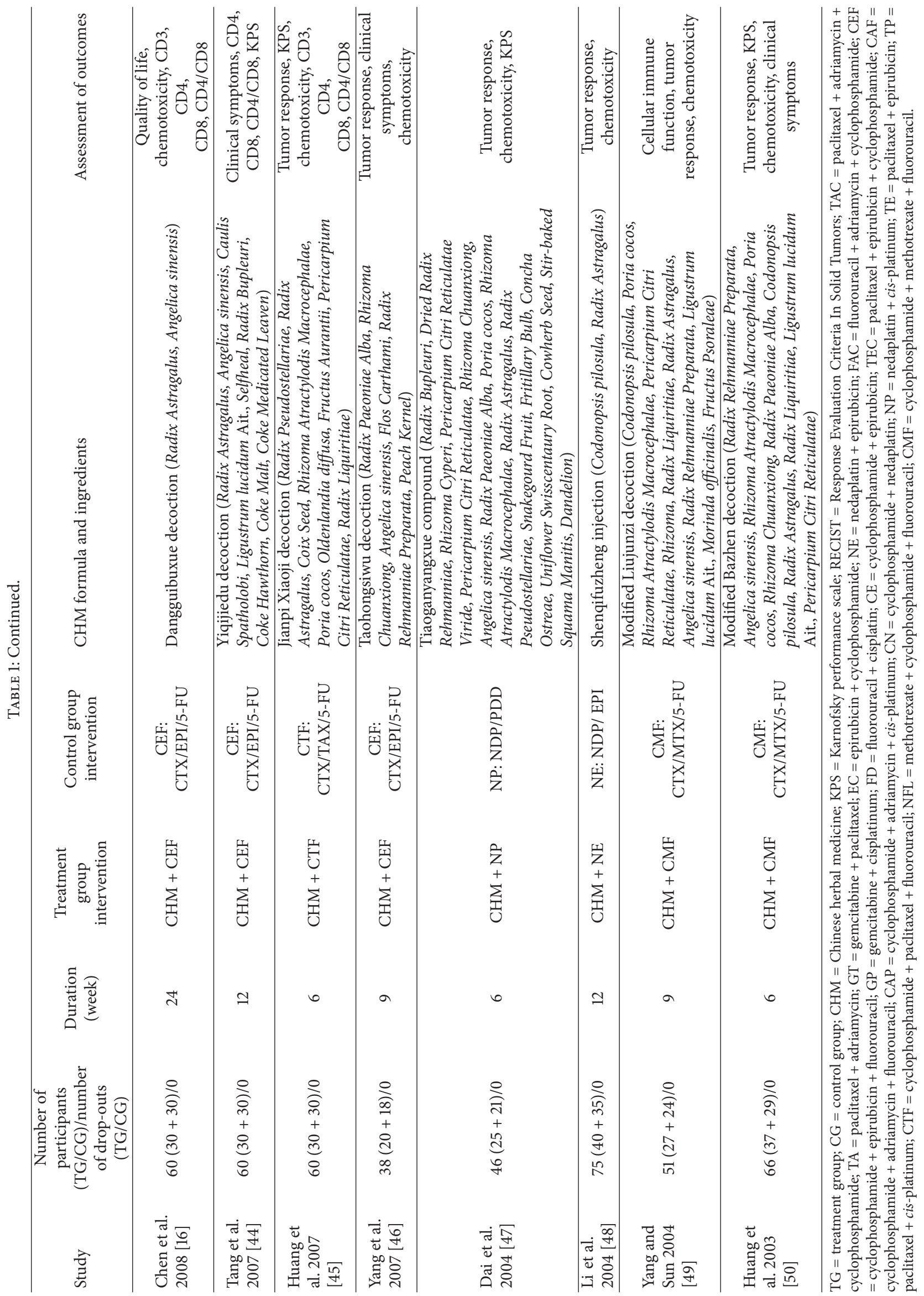




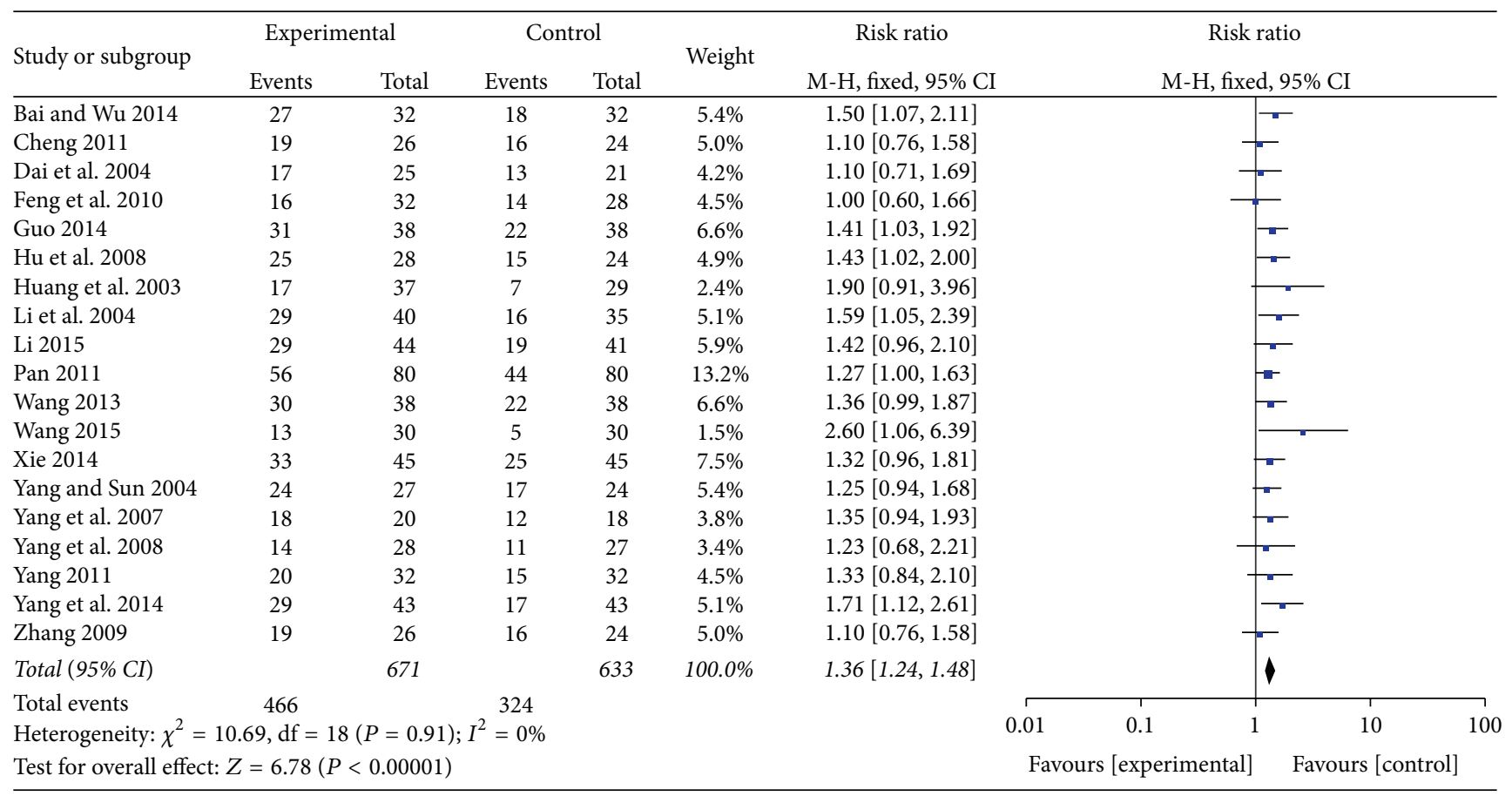

(a) Tumor response $\mathrm{CR}+\mathrm{PR}$

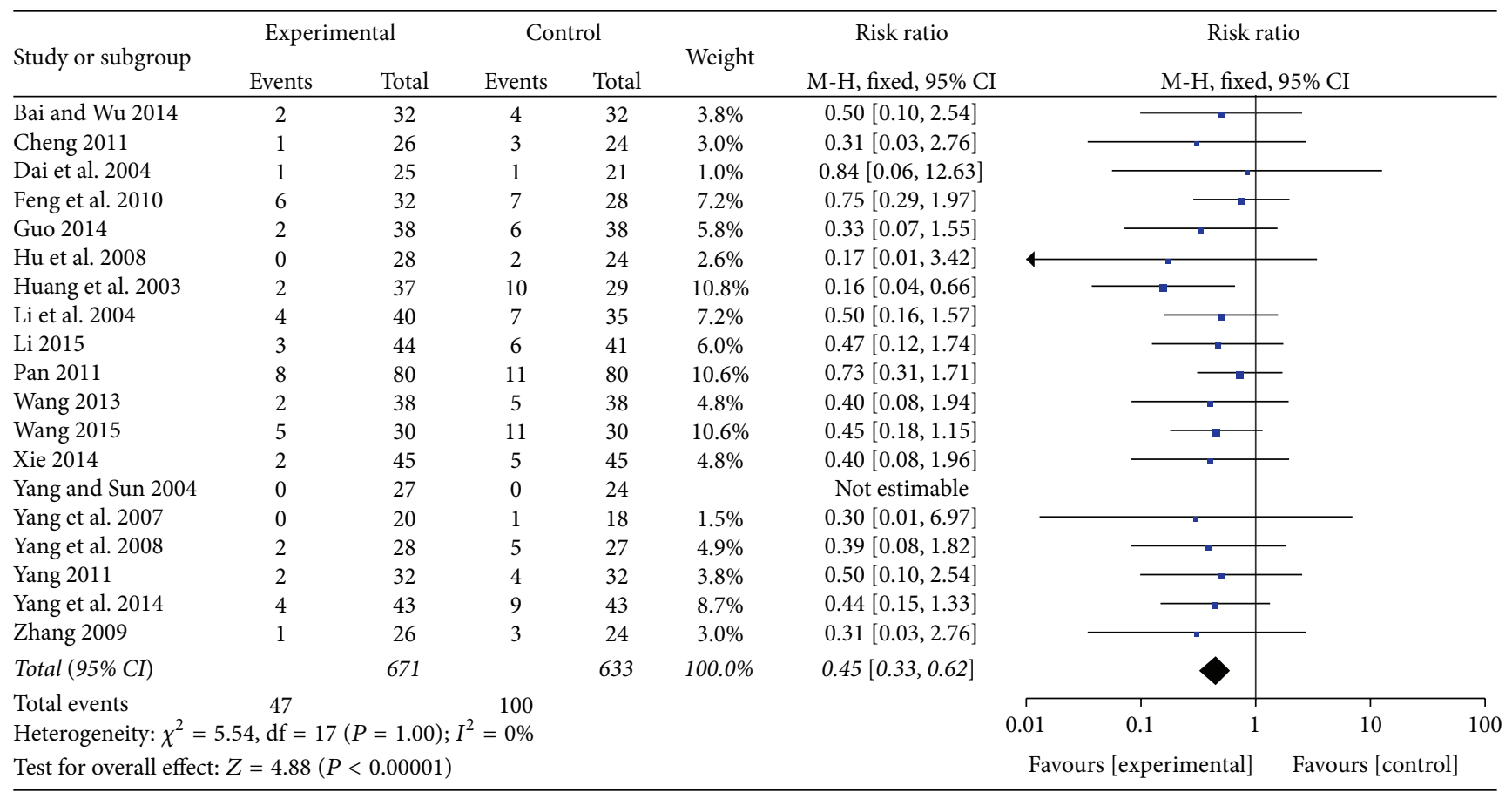

(b) Tumor response PD

FIGURE 4: Tumor response.

cyclophosphamide that is a common used chemotherapeutic medicine [63]. Although the mechanism of the anticancer effects of most CHMs is not fully understood, the effect of stimulating the immune system and reducing the toxicity induced by chemotherapy might be the two major advantages of $\mathrm{CHM}$ as an adjunctive therapy in the treatment of breast cancer.

Tumor response, performance status, toxicity induced by chemotherapy, and immunoregulation were the four major outcome measurements in the study. However, not 


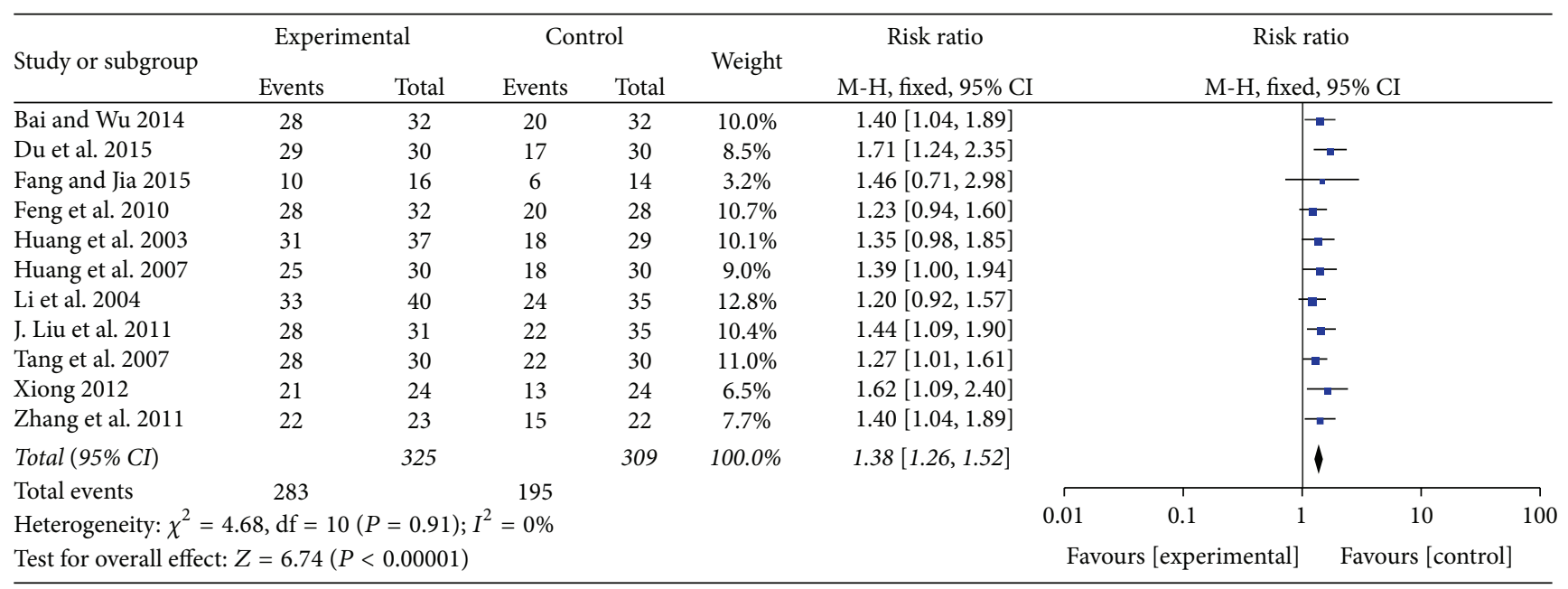

(a) Number of patients with nondeterioration KPS

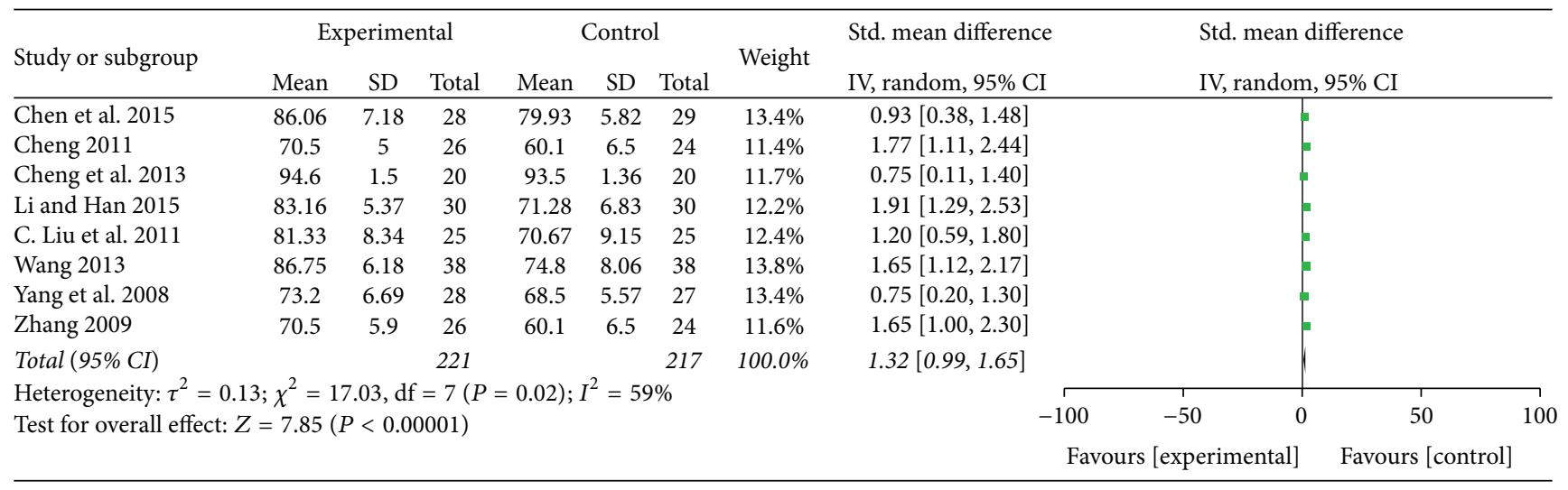

(b) KPS (mean \pm SD)

FIGURE 5: Quality of life: KPS.

all included studies simultaneously reported all the four outcomes. For example, Li and Han [52] only reported tumor response and chemotoxicity while Huang et al. [45] reported all four outcomes. Despite that, all available data in these studies were analyzed without any subjective selection. CD3 T cell level, CD4 T cell level, CD8 T cell level, and CD4/CD8 ratio are used as measurements for the evaluation of immunoregulation in breast cancer patients. In this study, we analyzed the mean value of CD3, CD4, CD8, and CD4/CD8 ratio between combined therapy group and chemotherapy group. Although results of these measurements showed that there was a significant improvement in patients treated by chemotherapy combined with CHM, the heterogeneity was also significant when we pooled individual studies which might be caused by different chemotherapy regimens used in different studies $[16,25,38,39,45,48,54]$. Hence, the efficacy of combined therapy in different chemotherapy regimens compared with chemotherapy alone was further analyzed. Patients treated by CHM combined with CTF (CTX/TAX/5$\mathrm{FU}$ ) reported a significant rise of $\mathrm{CD} 3 \mathrm{~T}$ cell level as compared with CTF alone $(\mathrm{MD}=6.53 ; 95 \% \mathrm{CI}=4.80-8.26 ; \mathrm{P}<$ $0.00001 ; I^{2}=13 \% ; 2$ studies; 105 patients). For CD4 T cell level, CD8 T cell level, and CD4/CD8 ratio, CHM combined with CTF and CHM combined with CEF (CTX/EPI/5-FU) both reported a significant improvement, while being with a contradictory result of heterogeneity test. For example, in the comparison of CD4 T cell level, the value of $I^{2}$ in patients treated by CHM combined with CEF was $0 \%$, while that in patients treated by CHM combined with CTF reported was $68 \%$. Similar situations also happened in CD8 T cell level and CD4/CD8 ratio. The variation of heterogeneity test in these studies may be due to the difference of treatment duration and therapeutic dose except that the different chemotherapy regimens are used in different studies.

The study protocol was registered in PROSPERO (International Prospective Register of Systematic Reviews) with registration number CRD42016033965. Although all reviewers in the study received high-quality training of systematic review and we strictly followed the review procedure stated by the Cochrane Collaboration, there were still several limitations in the study. Firstly, most of the included studies did not clearly describe allocation concealment and blinding, which may contribute to overestimate the effect of treatment group and the emergence of bias. Secondly, publication 


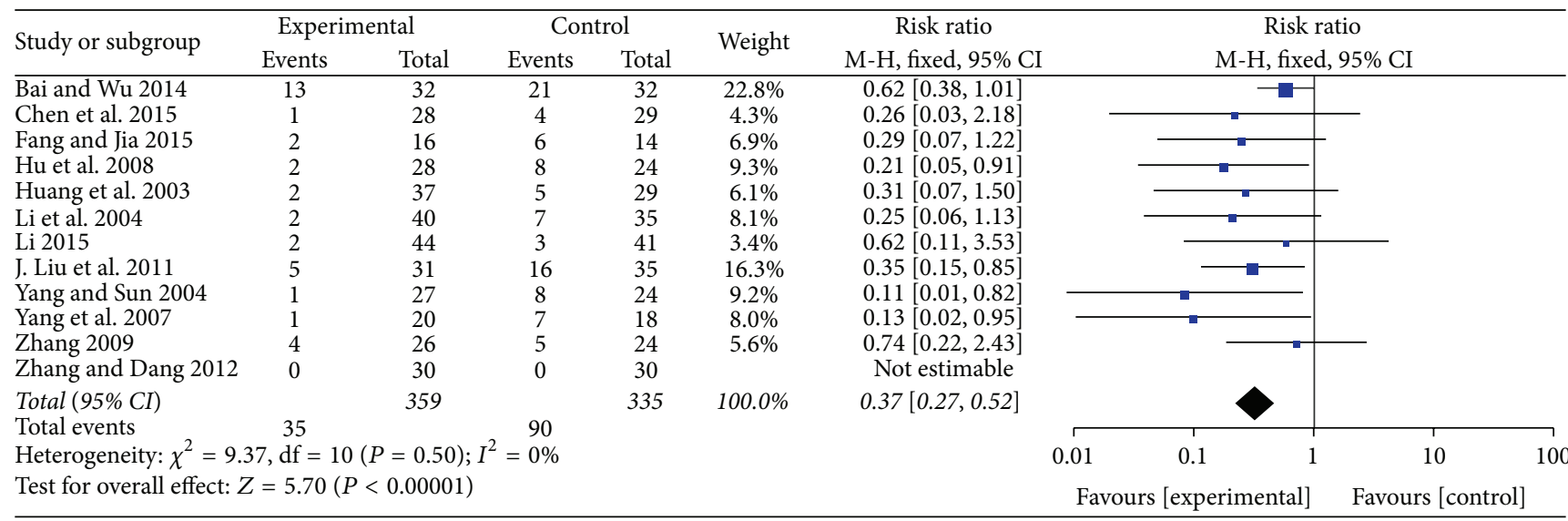

(a) Nausea and vomiting at toxicity grade of III-IV

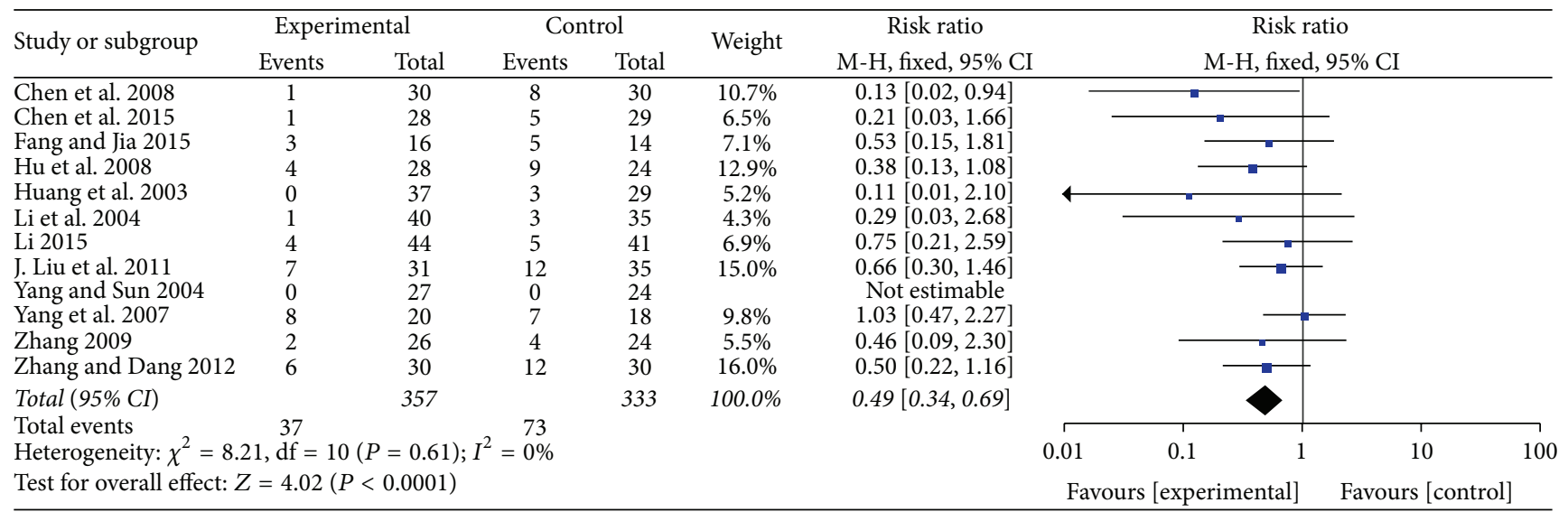

(b) WBC reduction at toxicity grade of III-IV

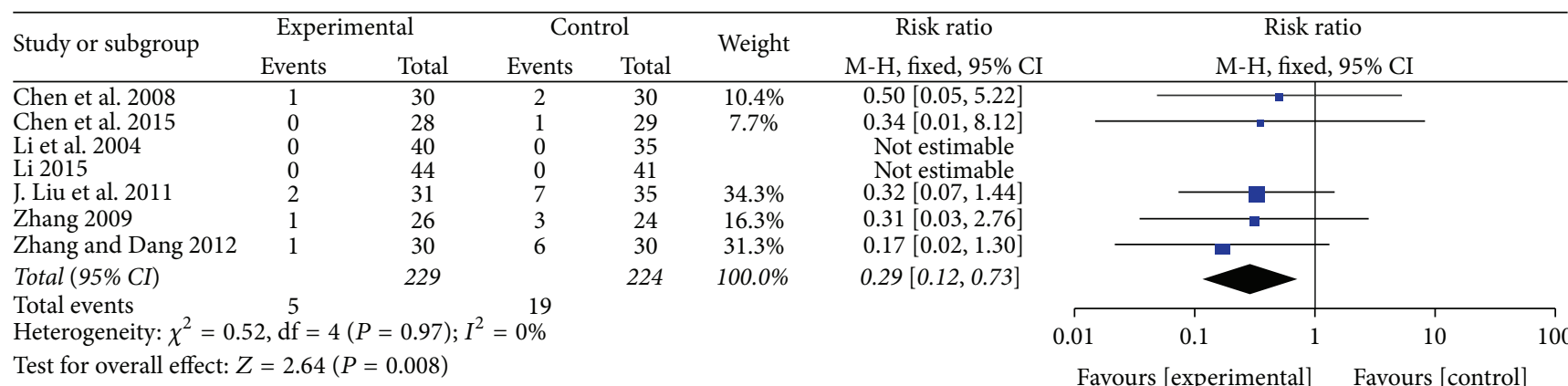

(c) Platelet reduction at toxicity grade of III-IV

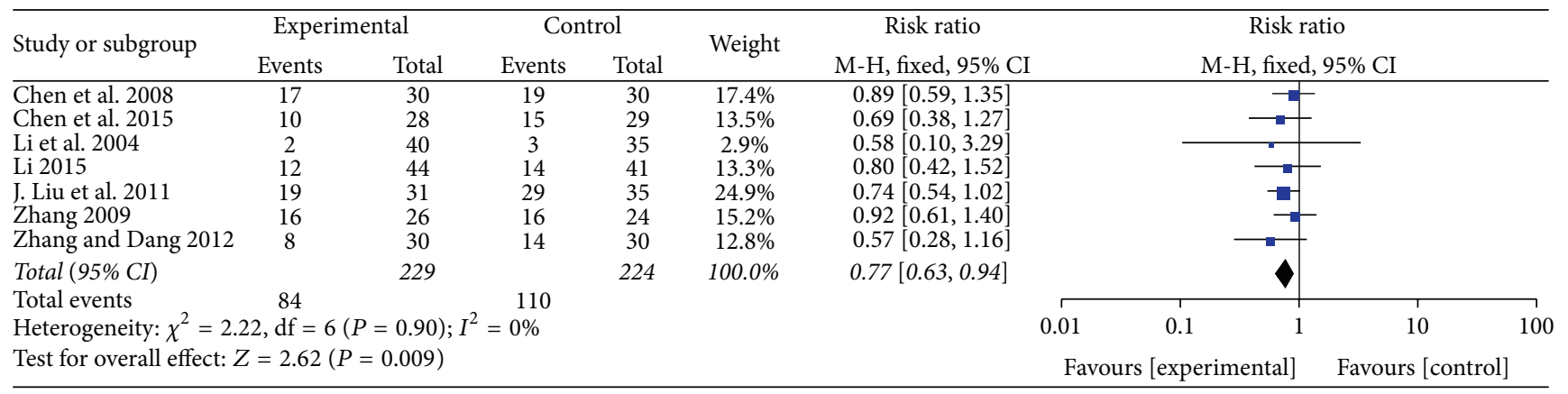

(d) Platelet reduction at toxicity grade of I-IV

FIGURE 6: Reduction of adverse effects. 


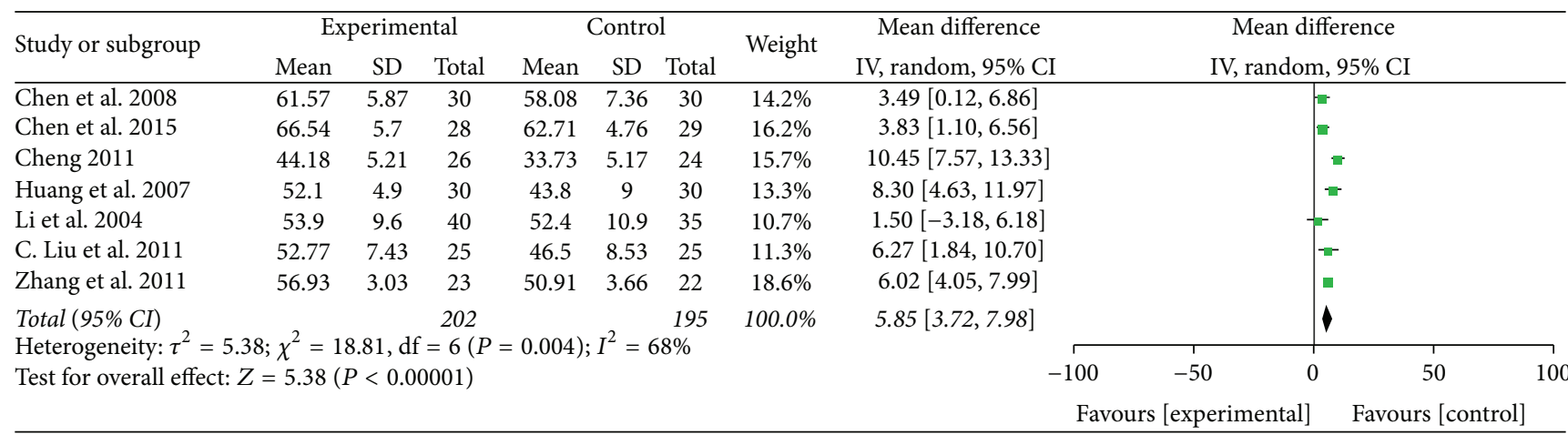

(a) $\mathrm{CD} 3$

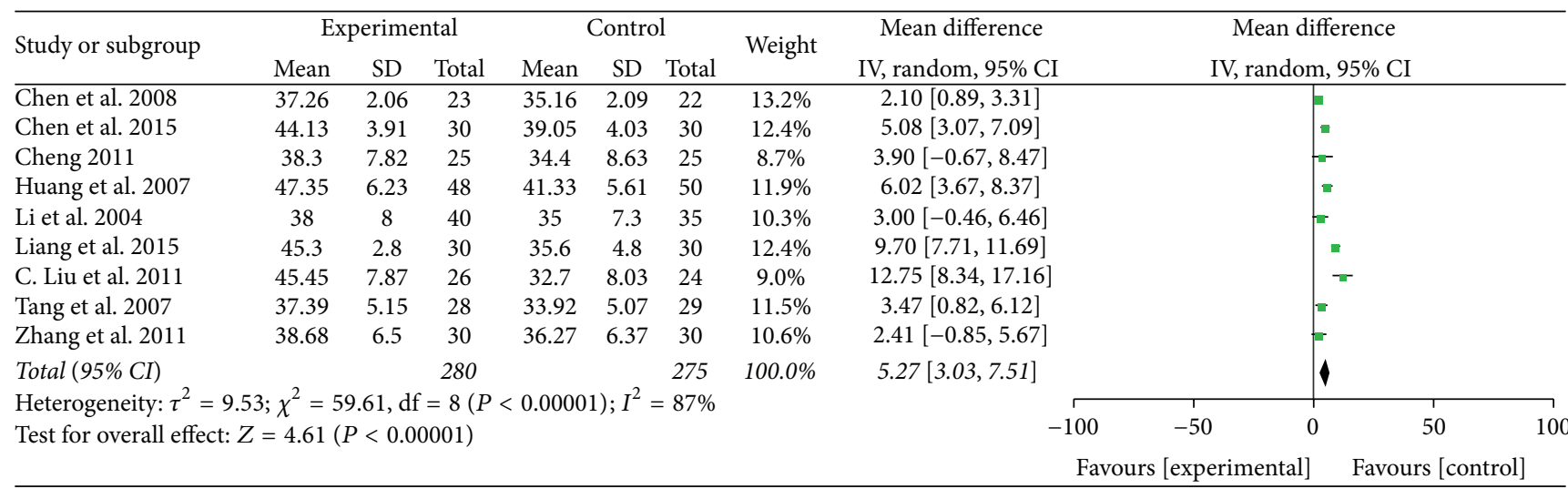

(b) $\mathrm{CD} 4$

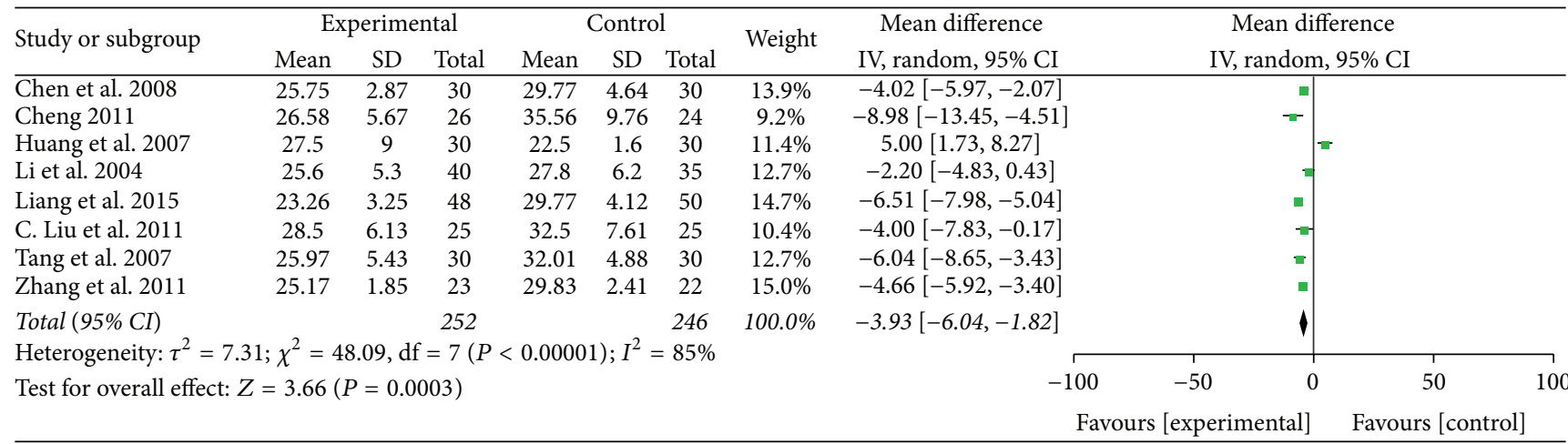

(c) $\mathrm{CD} 8$

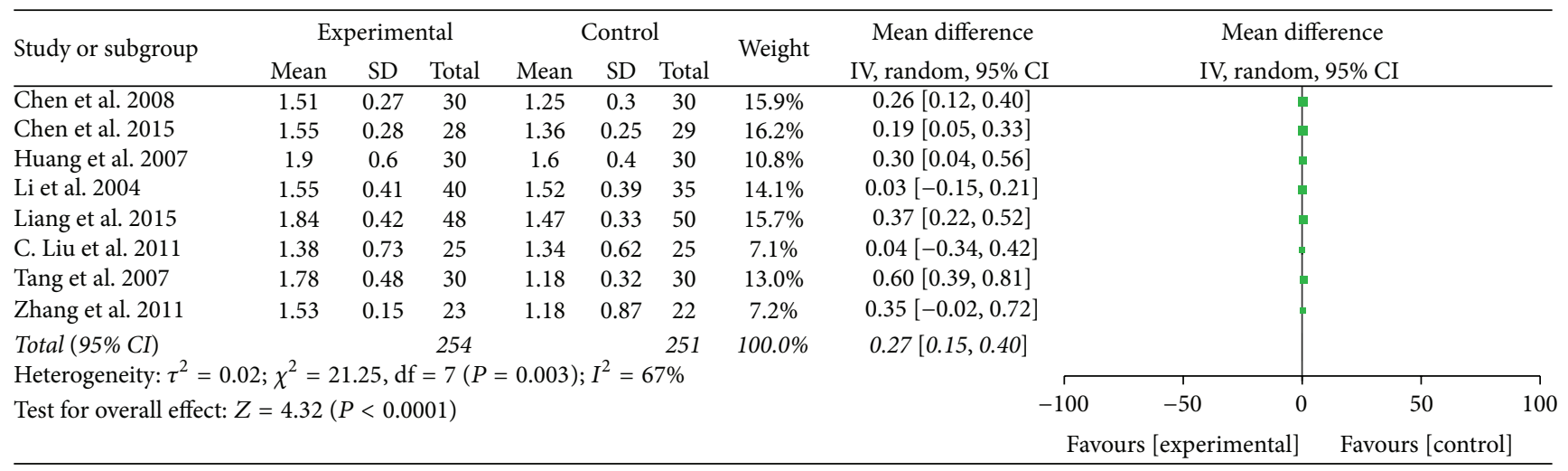

(d) $\mathrm{CD} 4 / \mathrm{CD} 8$ ratio

FiguRE 7: Immunoregulation. 
TABLE 2: Herbs frequently used for breast cancer.

\begin{tabular}{lcc}
\hline \multirow{2}{*}{ Chinese herbal medicine } & \multicolumn{2}{c}{ Frequency } \\
& Count & $\%$ \\
\hline Radix Astragalus & 24 & $73 \%$ \\
Rhizoma Atractylodis Macrocephalae & 20 & $61 \%$ \\
Angelica sinensis & 16 & $48 \%$ \\
Codonopsis pilosula & 15 & $45 \%$ \\
Poria cocos & 13 & $39 \%$ \\
Radix Liquiritiae & 12 & $36 \%$ \\
Pericarpium Citri Reticulatae & 11 & $33 \%$ \\
Radix Paeoniae Alba & 9 & $27 \%$ \\
Coix Seed & 8 & $24 \%$ \\
Radix Bupleuri & 7 & $21 \%$ \\
\hline
\end{tabular}

bias may exist in the study because almost all included studies reported the positive results, while some negative results may be selectively unreported and therefore were not included in the present systematic review. Thirdly, most of the included studies did not provide enough information about methodology such as the way to generate random sequence, intention-to-treat analysis, follow-up, and drop-out rate; the methodological flaws may lead to potential biases. Lastly, different interventions are used in the studies such as different chemotherapy regimens, CHM composition (single herb of compound), oral administration and intravenous injection, treatment duration, or dosage, and all these may lead to heterogeneity among the studies.

\section{Conclusion}

The evidence from this systematic review shows that using CHM as an adjuvant therapy to chemotherapy in comparison with chemotherapy alone has advantages in breast cancer patients. However, particular attention should be paid for applying appropriate and scientific research methodologies to explore CHM as a holistic system because of the complex nature of CHM interventions [64]. Moreover, due to the small sample size, the findings of this meta-analysis may not apply to all patients with breast cancer. Therefore more RCTs with high-quality and large scale are worth performing to investigate the other potential interest of CHM as adjuvant therapy in breast cancer patients, such as survival rate, recurrence rate, and local and distant metastasis, shorten the course of chemotherapy, and so forth.

\section{Competing Interests}

All authors declare that they have no potential competing interests.

\section{Authors' Contributions}

Libing Zhu and Lingru Li contributed equally to this work. Libing Zhu and Lingru Li retrieved and analyzed data and wrote and revised this paper. Yingshuai Li retrieved data.
Ji Wang conceived and supervised the study. Qi Wang interpreted data and edited the paper.

\section{Acknowledgments}

This work was supported by the National Key Basic Research and Development Program (973 Program) (no. 2011CB505400).

\section{References}

[1] D. M. Parkin, "Global cancer statistics in the year 2000," The Lancet Oncology, vol. 2, no. 9, pp. 533-543, 2001.

[2] R. L. Siegel, K. D. Miller, and A. Jemal, "Cancer statistics, 2016," CA: A Cancer Journal for Clinicians, vol. 66, no. 1, pp. 7-30, 2016.

[3] C. Sotiriou, S.-Y. Neo, L. M. McShane et al., "Breast cancer classification and prognosis based on gene expression profiles from a population-based study," Proceedings of the National Academy of Sciences of the United States of America, vol. 100, no. 18, pp. 10393-10398, 2003.

[4] T. Sørlie, C. M. Perou, R. Tibshirani et al., "Gene expression patterns of breast carcinomas distinguish tumor subclasses with clinical implications," Proceedings of the National Academy of Sciences of the United States of America, vol. 98, no. 19, pp. 1086910874, 2001.

[5] Y. Cui, X.-O. Shu, Y. Gao et al., "Use of complementary and alternative medicine by Chinese women with breast cancer," Breast Cancer Research and Treatment, vol. 85, no. 3, pp. 263270, 2004.

[6] J. J. Tao, K. Visvanathan, and A. C. Wolff, "Long term side effects of adjuvant chemotherapy in patients with early breast cancer," The Breast, vol. 24, supplement 2, pp. S149-S153, 2015.

[7] A. M. Gonzalez-Angulo, F. Morales-Vasquez, and G. N. Hortobagyi, "Overview of resistance to systemic therapy in patients with breast cancer," Advances in Experimental Medicine and Biology, vol. 608, pp. 1-22, 2007.

[8] A. Molassiotis, J. A. Scott, N. Kearney et al., "Complementary and alternative medicine use in breast cancer patients in Europe," Supportive Care in Cancer, vol. 14, no. 3, pp. 260-267, 2006.

[9] A. Molassiotis, P. Fernandez-Ortega, D. Pud et al., "Use of complementary and alternative medicine in cancer patients: a European survey," Annals of Oncology, vol. 16, no. 4, pp. 655663, 2005.

[10] J. Shen, R. Andersen, P. S. Albert et al., "Use of complementary/alternative therapies by women with advanced-stage breast cancer," BMC Complementary and Alternative Medicine, vol. 2, article 8, 2002.

[11] L. M. DiGianni, J. E. Garber, and E. P. Winer, "Complementary and alternative medicine use among women with breast cancer," Journal of Clinical Oncology, vol. 20, no. 18, supplement, pp. 34s38s, 2002.

[12] J. Huebner, K. Muenstedt, F. J. Prott et al., "Online survey of patients with breast cancer on complementary and alternative medicine," Breast Care, vol. 9, no. 1, pp. 60-63, 2014.

[13] A. Sparber, L. Bauer, G. Curt et al., "Use of complementary medicine by adult patients participating in cancer clinical trials," Oncology Nursing Forum, vol. 27, no. 4, pp. 623-630, 2000.

[14] Z. Chen, K. Gu, Y. Zheng, W. Zheng, W. Lu, and X. O. Shu, "The use of complementary and alternative medicine among 
Chinese women with breast cancer," Journal of Alternative and Complementary Medicine, vol. 14, no. 8, pp. 1049-1055, 2008.

[15] W. C. S. Cho, "Scientific evidence on the supportive cancer care with chinese medicine," Chinese Journal of Lung Cancer, vol. 13, no. 3, pp. 190-194, 2010.

[16] Q. T. Chen, H. X. Zhu, Z. Q. Liu, H. B. Wang, Y. Xu, and Y. $\mathrm{Li}$, "Clinical observation on the effect-enhancing and toxicityreducing efficacy of Dangguibuxue decoction for postoperative breast cancer patients with chemotoxicity," Journal of Hebei Medical College for Continuing Education, no. 6, pp. 50-51, 2008.

[17] Y. Q. Liang, W. J. Yin, W. Qian, H. L. Wang, C. Ma, and H. Y. Du, "Effects of Huaier Granule combined with systemic chemotherapy on immunologic function and prognosis for advanced breast cancer patients," Chinese Journal of Bases and Clinics in General Surgery, no. 12, pp. 1482-1486, 2015.

[18] Y. L. Du, Z. M. Wang, F. Wang, and D. Y. Lu, "Efficacy observation on modified Xiaoyao powder combined with chemotherapy for postoperative breast cancer," Modern Journal of Integrated Traditional Chinese Medicine and Western Medicine, no. 3, pp. 295-297, 2015.

[19] A. B. Miller, B. Hoogstraten, M. Staquet, and A. Winkler, "Reporting results of cancer treatment," Cancer, vol. 47, no. 1, pp. 207-214, 1981.

[20] L. L. D. Zhong, H.-Y. Chen, W. C. S. Cho, X.-M. Meng, and Y. Tong, "The efficacy of Chinese herbal medicine as an adjunctive therapy for colorectal cancer: a systematic review and metaanalysis," Complementary Therapies in Medicine, vol. 20, no. 4, pp. 240-252, 2012.

[21] WHO, WHO Handbook for Reporting Results of Cancer Treatment, World Health Organization, Geneva, Switzerland, 1979.

[22] J. Higgins and S. Green, Cochrane Handbook for Systematic Reviews of Interventions, Version 5.1.0, 2016.

[23] J. P. T. Higgins and S. Green, Cochrane Handbook for Systematic Reviews of Interventions, Version 5.1.0, 2011.

[24] H. W. Li, "Self-made Fuzheng Xiaoliufang as an adjuvant therapy for advanced breast cancer and its impact on serum tumor marker," China Pharmaceuticals, no. 3, pp. 11-13, 2015.

[25] H. H. Chen, W. Wang, G. X. Shi, and J. W. Li, "Effect of Chinese herbal compound combining chemotherapy on recurrence breast cancer," Liaoning Journal of Traditional Chinese Medicine, no. 5, pp. 1003-1005, 2015.

[26] J. G. Wang, “The long-term efficacy observation on Xiaozheng compound as an adjuvant therapy for breast cancer liver metastasis," Journal of New Chinese Medicine, no. 6, pp. 224225, 2015.

[27] L. L. Fang and Y. Jia, "Method of promoting blood circulation to remove blood stasis and detoxication combined with chemotherapy on breast cancer of intermediate stage and advanced stage," Journal of Shanxi College of Traditional Chinese Medicine, no. 2, pp. 52-53, 2015.

[28] J. W. Bai and W. M. Wu, "Efficacy analysis of Jin Long Capsule (JLC) in neoadjuvant chemotherapy of breast cancer," Chinese Journal of Clinical Oncology, no. 4, pp. 246-249, 2014.

[29] L. Guo, "Experience of 76 cases with mammary cancer treated with integrated Chinese and western medicine," Clinical Journal of Chinese Medicine, no. 15, pp. 137-138, 2014.

[30] F. Xie, "Clinical analysis Shenqifuzheng injection combined with chemotherpay in the treatment of breast cancer," Guide of China Medicine, no. 30, pp. 19-20, 2014.

[31] J. Yang, J. L. He, X. X. Li, and Y. H. Yang, "Analysis of curative effect of spleen and kidney strengthening herb combined with observation of 43 cases," Guiding Journal of Traditional Chinese Medicine and Pharmacy, no. 13, pp. 33-35, 2014.

[32] Y. S. Cheng, F. Y. Gong, A. W. Li, Y. S. Wen, G. J. Wang, and Y. Lv, "Modified Lizhong decoction for nausea and vomiting caused by breast cancer chemotherapy," Journal of Sichuan of Traditional Chinese Medicine, no. 5, pp. 90-91, 2013.

[33] D. J. Wang, "Clinical observation of Chinese and Western drugs for the treatment of 76 cases of breast cancer," Medical Recapitulate, vol. 14, pp. 2676-2678, 2013.

[34] L. K. Xiong, "The impact of Fuzheng Xiaoji compound combined with chemotherapy on the quality of life and serum tumor marker of breast cancer patients," Yunnan Journal of Traditional Chinese Medicine, no. 4, pp. 14-15, 2012.

[35] S. X. Zhang and H. Dang, "The method of Fuzheng Guben combined with NP chemotherapy for 30 cases advanced breast cancer," Guangming Journal of Chinese Medicine, no. 5, pp. 980982, 2012.

[36] L. M. Pan, "Cinobufagin combined with chemotherapy for 160 cases advanced breast cancer," China Foreign Medical Treatment, no. 1, pp. 59-61, 2011.

[37] G. W. Yang, "Combination of the magical power of trichosanthes and chemotherapy in advanced breast cancer," Journal of Liaoning University of TCM, no. 7, pp. 84-85, 2011.

[38] Y. Cheng, "Clinical observation on Fuzheng Kanai compound combined TP for advanced breast cancer," Medical Technology, no. 12, pp. 92-93, 2011.

[39] Y. C. Zhang, X. J. Li, Y. J. Jia, J. Chen, and Y. Y. Sun, “Clinical observation of 'Fuzheng Quyu Jiedu Prescription' combining with chemotherapy in treating postoperative breast cancer," Shanghai Journal of Traditional Chinese Medicine, no. 11, pp. 6466, 2011.

[40] J. J. Feng, J. P. Zhao, W. K. Jia, G. Y. Zhao, and T. Peng, “Effect observation of chemotherapy with modified Wumeiwan on advanced breast cancer," Shanxi Journal of Traditional Chinese Medicine, no. 7, pp. 33-34, 2010.

[41] P. Y. Zhang, "Chinese herbal compound combined with chemotherapy for 50 cases advanced breast cancer," Liaoning Journal of Traditional Chinese Medicine, no. 4, pp. 130-131, 2009.

[42] G. W. Yang, Y. M. Xu, Q. Fu et al., "Clinical observat ion on guben Yiliu II combined with chemotherapy in the treatment of 28 cases of advanced breast cancer," Journal of Traditional Chinese Medicine, no. 12, pp. 1081-1083, 2008.

[43] J. H. Hu, Y. F. Yuan, and J. Q. He, "Yiqihuoxue decoction combined with new adjuvant chemotherapy for 28 cases breast cancer," Hunan Journal of Traditional Chinese Medicine, no. 2, pp. 38-39, 2008.

[44] J. W. Tang, L. Li, and X. Y. Tang, "Yiqijiedu decoction combined with chemotherapy for 30 cases breast cancer (type Qi-deficiency while toxin flourishing)," Traditional Chinese Medicine Research, no. 7, pp. 28-29, 2007.

[45] Z. F. Huang, J. B. Liu, Q. S. Chen, H. Z. Li, Z. J. Zhang, and C. J. Huang, "The effect of Jianpi Xiaoji decoction combined with chemotherapy for the quality of life and immunologic function of advanced breast cancer patients," Journal of New Chinese Medicine, no. 5, pp. 88-89, 2007.

[46] H. Y. Yang, C. L. Tong, and M. Huang, "Clinical observation on Taohongsiwu decoction combined with new adjuvant chemotherapy for breast cancer with stagnation of blood stasis," Modern Journal of Integrated Traditional Chinese and Western Medicine, no. 10, pp. 1327-1328, 2007. 
[47] H. Dai, C. L. Zhu, and X. L. Wang, "Tiaoganyangxue decoction combined with NP chemotherapy for 25 cases advanced breast cancer," Jilin Journal of Traditional Chinese Medicine, no. 4, pp. 33-34, 2004.

[48] X. L. Li, Q. Y. Tian, and W. J. Ma, "Clinical observation on Senqifuzheng injection combined with chemotherpay for advanced breast cancer," Modern Oncology, no. 6, pp. 574-575, 2004.

[49] L. Yang and S. Sun, "Clinical research into the treatment of mammary cancer by chemotherapy combined with TCM drugs before operation," Journal of Hennan University of Chinese Medicine, no. 4, pp. 40-41, 2004.

[50] Z. F. Huang, J. S. Wei, Z. Y. Shi, and K. Zhong, "Study of Bazhen decoction and chemotherapy on mammary cancer in metaphase or later period," Modern Journal of Integr ated Traditional Chinese and Western Medicine, no. 11, pp. 1123-1124, 2003.

[51] T. Zhou and F. J. Zhou, "Gonghuanyangxue granule for the Qiblood-deficiency syndrome of breast cancer after new adjuvant chemotherapy," Guiding Journal of Traditional Chinese Medicine and Pharmacy, no. 6, pp. 63-65, 2015.

[52] L. F. Li and M. Han, "The effect of YIqihuoxue method as an adjuvant therapy for postoperative breast cancer and its impact on patients' quality of life," Sichuan Jouranl of Traditional Chinese Medicine, no. 3, pp. 119-121, 2015.

[53] J. Liu, S. X. Wei, and M. Lu, “The observation of attenuation and synergy of postoperative chemotherapy matching with traditional Chinese drug Tianzhicao capsule in breast cancer," Medical Journal of West China, no. 5, pp. 896-897, 2011.

[54] C. Liu, L. Y. Wu, H. J. Zhao, and N. Yang, "Clinical observation on Rensenyangrong decoction for the Qi-blood-deficiency type of breast cancer which caused by new adjuvant chemotherapy," The Western Journal of Traditional Chinese Medicine, no. 11, pp. 8-11, 2011.

[55] K. H. Chang, R. Brodie, M. A. Choong, K. J. Sweeney, and M. J. Kerin, "Complementary and alternative medicine use in oncology: a questionnaire survey of patients and health care professionals," BMC Cancer, vol. 11, article 196, 2011.

[56] S. G. Li, H. Y. Chen, C. S. Ou-Yang et al., "The efficacy of Chinese herbal medicine as an adjunctive therapy for advanced nonsmall cell lung cancer: a systematic review and meta-analysis," PLoS ONE, vol. 8, no. 2, Article ID e57604, 2013.

[57] W. C. S. Cho and H.-Y. Chen, "Clinical efficacy of traditional Chinese medicine as a concomitant therapy for nasopharyngeal carcinoma: a systematic review and meta-analysis," Cancer Investigation, vol. 27, no. 3, pp. 334-344, 2009.

[58] W. C. S. Cho and H.-Y. Chen, "Transcatheter arterial chemoembolization combined with or without Chinese herbal therapy for hepatocellular carcinoma: meta-analysis," Expert Opinion on Investigational Drugs, vol. 18, no. 5, pp. 617-635, 2009.

[59] J. S. Yates, K. M. Mustian, G. R. Morrow et al., "Prevalence of complementary and alternative medicine use in cancer patients during treatment," Supportive Care in Cancer, vol. 13, no. 10, pp. 806-811, 2005.

[60] K. I. Block and M. N. Mead, "Immune system effects of echinacea, ginseng, and astragalus: a review," Integrative Cancer Therapies, vol. 2, no. 3, pp. 247-267, 2003.

[61] W. C. S. Cho and K. N. Leung, "In vitro and in vivo immunomodulating and immunorestorative effects of Astragalus membranaceus," Journal of Ethnopharmacology, vol. 113, no. 1, pp. 132-141, 2007.
[62] M. McCulloch, C. See, X.-J. Shu et al., "Astragalus-based Chinese herbs and platinum-based chemotherapy for advanced non-small-cell lung cancer: meta-analysis of randomized trials," Journal of Clinical Oncology, vol. 24, no. 3, pp. 419-430, 2006.

[63] W. Kim, S.-H. Kim, S. K. Park, and M. S. Chang, "Astragalus membranaceus ameliorates reproductive toxicity induced by cyclophosphamide in male mice," Phytotherapy Research, vol. 26, no. 9, pp. 1418-1421, 2012.

[64] A. Flower, C. Witt, J. P. Liu, G. Ulrich-Merzenich, H. Yu, and G. Lewith, "Guidelines for randomised controlled trials investigating Chinese herbal medicine," Journal of Ethnopharmacology, vol. 140, no. 3, pp. 550-554, 2012. 


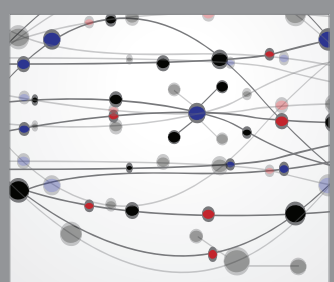

The Scientific World Journal
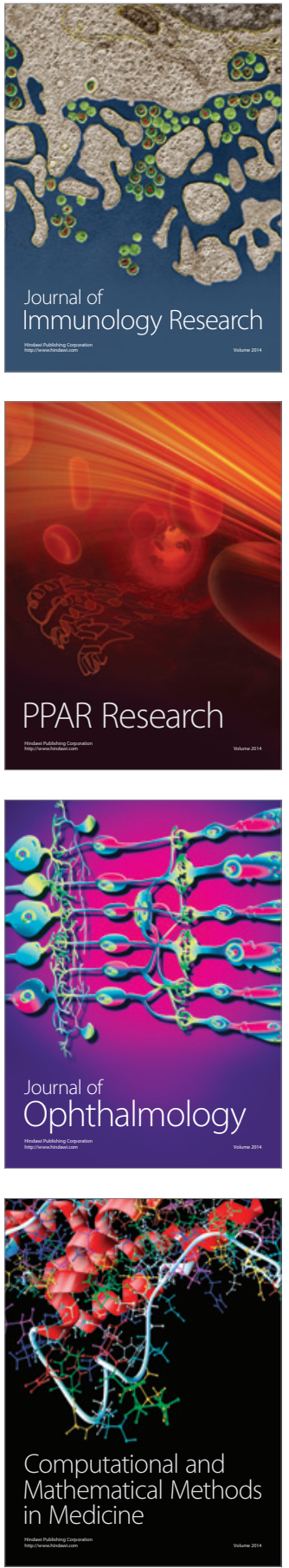

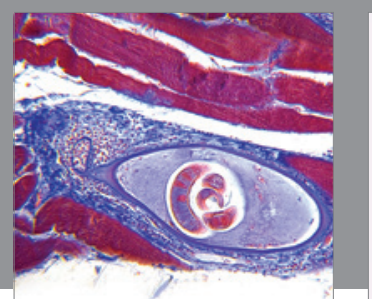

Gastroenterology Research and Practice

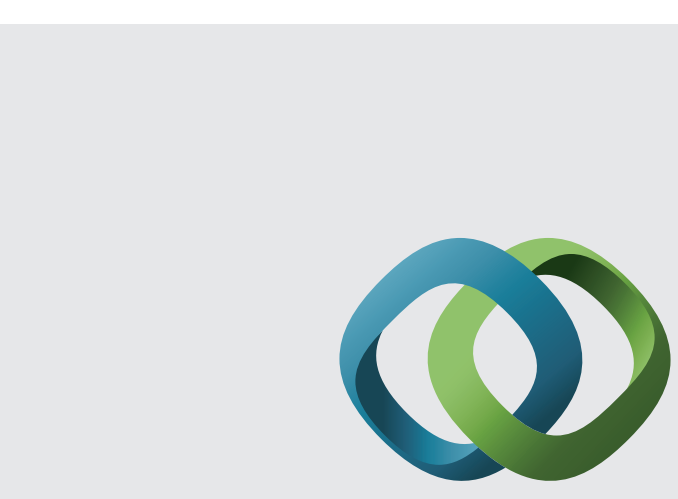

\section{Hindawi}

Submit your manuscripts at

http://www.hindawi.com
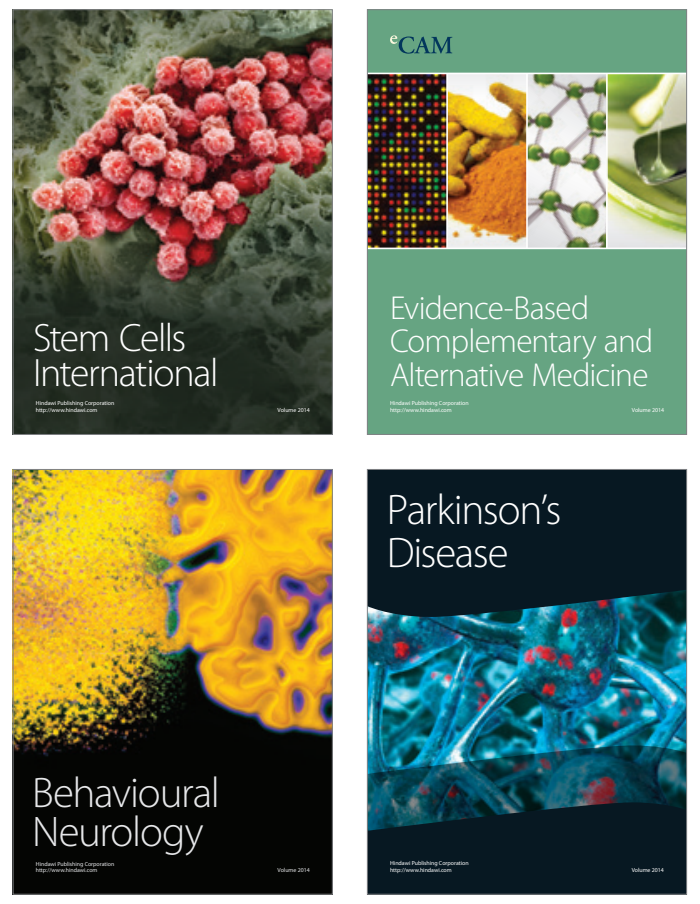
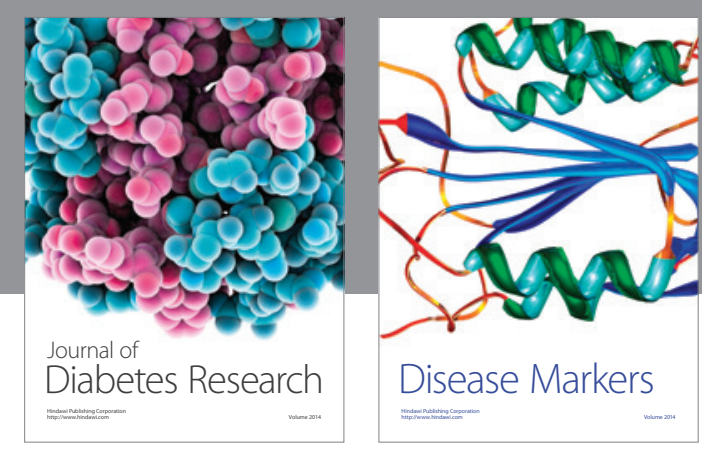

Disease Markers
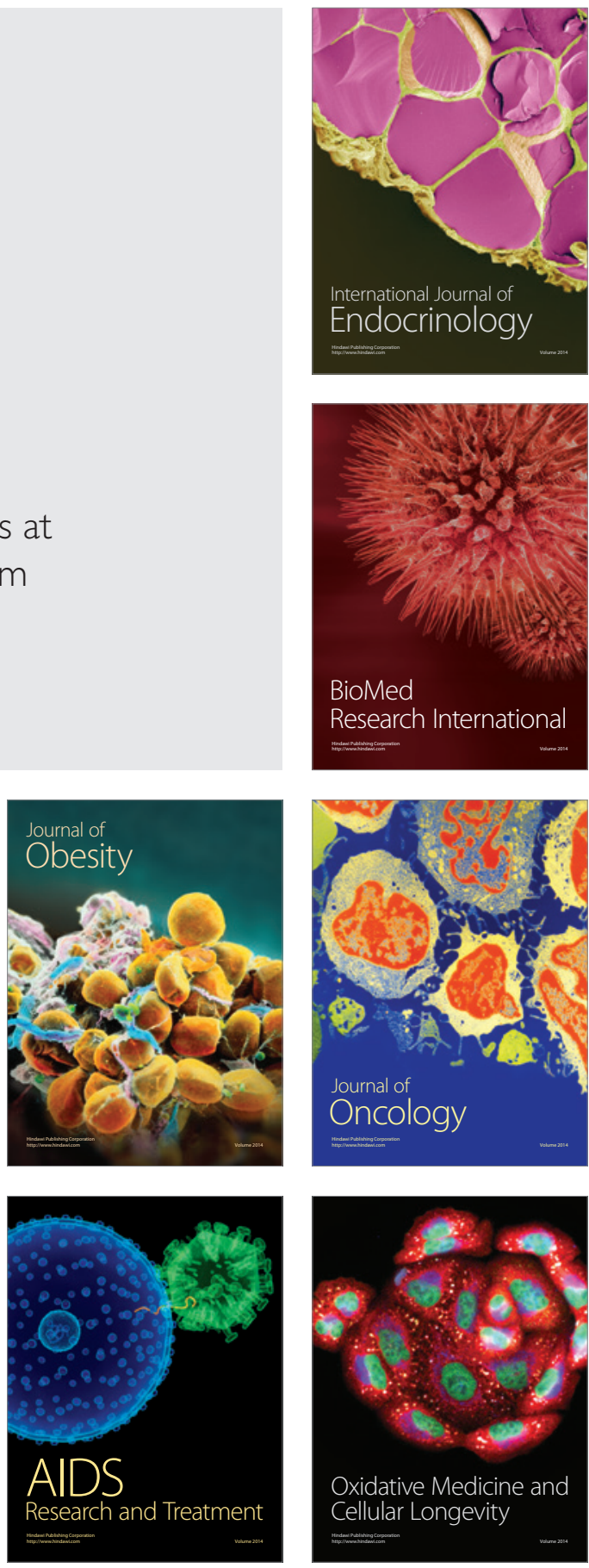